\title{
Collaborative Spectrum Sensing Optimisation Algorithms for Cognitive Radio Networks
}

\author{
Kamran Arshad, Muhammad Ali Imran, and Klaus Moessner \\ Centre for Communication Systems Research, University of Surrey, Guildford GU2 7XH, UK \\ Correspondence should be addressed to Kamran Arshad, k.arshad@surrey.ac.uk
}

Received 28 October 2009; Accepted 17 March 2010

Academic Editor: Massimiliano Laddomada

Copyright ( $) 2010$ Kamran Arshad et al. This is an open access article distributed under the Creative Commons Attribution License, which permits unrestricted use, distribution, and reproduction in any medium, provided the original work is properly cited.

\begin{abstract}
The main challenge for a cognitive radio is to detect the existence of primary users reliably in order to minimise the interference to licensed communications. Hence, spectrum sensing is a most important requirement of a cognitive radio. However, due to the channel uncertainties, local observations are not reliable and collaboration among users is required. Selection of fusion rule at a common receiver has a direct impact on the overall spectrum sensing performance. In this paper, optimisation of collaborative spectrum sensing in terms of optimum decision fusion is studied for hard and soft decision combining. It is concluded that for optimum fusion, the fusion centre must incorporate signal-to-noise ratio values of cognitive users and the channel conditions. A genetic algorithm-based weighted optimisation strategy is presented for the case of soft decision combining. Numerical results show that the proposed optimised collaborative spectrum sensing schemes give better spectrum sensing performance.
\end{abstract}

\section{Introduction}

As numbers of wireless devices, innovative services, and number of mobile users continue to grow, more and more spectrum resources will be needed to guarantee desired Quality of Service. Mobile users want high-quality calls, streaming videos, and high-speed downloads, placing more and more stress on the limited radio spectrum available to the network operators. The radio spectrum spans around 300 billion frequencies; however, only a tiny fraction of frequencies can be used for commercial or personal radio communications; fundamental physical limits apply [1]. In the current spectrum regulatory framework, most frequency bands are exclusively allocated to the privileged users, often called Primary User (PU), which have all the rights to use the allocated bands. This approach protects PU's from any intersystem interference, but on the other hand, it yields highly inefficient use of the spectrum.

Measurements conducted by the Office of Communications (Ofcom) in UK and the Spectrum Policy Task Force (SPTF) in USA indicate that many chunks of the licensed spectrum are not used or only partially used, for significant periods of time $[2,3]$. Spectrum occupancy measurements undertaken by Ofcom in Central London, at Heathrow airport and in some rural areas of the country, clearly show that there are significant portions of the radio spectrum which are not fully utilised in various geographical areas of the United Kingdom [4]. Similarly, in New York city maximum spectrum occupancy is reported as only $13.1 \%$ and downtown of Washington D.C. indicated spectrum occupancy of less than $35 \%$ of the radio spectrum below $3 \mathrm{GHz}$ [5]. These studies clearly suggest that currently spectrum scarcity is mainly due to the inefficient use of spectrum rather than the physical shortage of spectrum. Particularly in UK, Olympic Games 2012 put extra pressure on Ofcom to plan the efficient use of radio spectrum to satisfy over 10 million spectators, around 15,000 participants and about 20,000 media personnel in the UK who will beam live pictures and commentary all around the world. Moreover, emerging as well as some existing operators are faced with the difficult task to gain access to the radio spectrum to operate their services [6]. In addition, access to a block of spectrum is very expensive as seen when the five operators were licensed for the 3rd generation mobile systems in the UK at a cost of around $£ 22.5$ billion [7]. More 
recently (early 2009), the FCC spectrum auction in USA raised a record $\$ 19.9$ billion dollars [8].

Cognitive Radio (CR) is widely regarded as the technology which will increase spectrum utilisation significantly in the next generation wireless communication systems by implementing opportunistic spectrum sharing. Spectrum sensing is one of the most critical functionalities in a cognitive radio network; it allows the unauthorised users, called Secondary Users (SUs), to detect unused portions of the spectrum called "spectrum holes" and opportunistically utilise these spectrum holes without causing harmful interference to the PU. The main goal of spectrum sensing is to obtain awareness about the spectrum usage and the existence of the PU in a certain geographical area at a particular period of time. In order to evaluate the performance of spectrum sensing, two metrics are of great interest: probability of detection and the probability of false alarm. Probability of detection, $P_{d}$, determines the level of interference-protection provided to the PU while probability of false alarm, $P_{f}$, indicates percentage of spectrum holes falsely declared as occupied [9]. In the context of opportunistic spectrum access, $P_{d}$ must be higher than some predefined threshold while $P_{f}$ should be lower than some desired criteria or as minimum as possible.

To enhance the performance of spectrum sensing, many techniques are available in the literature, and a brief survey has been recently published in [10]. In practice, CRs usually have no or limited knowledge about the primary signals; hence the optimal spectrum sensing technique is energy detection [11]. An energy detection approach for spectrum sensing at an individual CR has been assumed in this paper because of its simplicity, ease of implementation, and low computational complexity [9]. Moreover, the aim of this paper is to characterise gains achieved by collaboration of users without going into the details of complex local spectrum sensing schemes. The more sophisticated techniques like match filter detection or cyclostationary feature detection can be used for signal classification if more a priori knowledge about the structure of the primary signal is available [10]. However, performance of the energy detector is susceptible to noise power uncertainty [12]. Nevertheless, it has been shown that Collaborative Spectrum Sensing (CSS) is capable of delivering the desired detection performance under noise uncertainty for a large number of users [9]. However, energy detectors do not work efficiently for detecting spread spectrum signals [10]; spread spectrum signals are out of the scope of this paper.

1.1. Prior Work. The spectrum scarcity and spectrum underutilisation problem has stimulated a number of exciting activities in the technical, economic, and regulatory domains in searching for better spectrum management policies and techniques, for example, FCC opened up some analogue TV bands for unlicensed access [13]. However, spectrum sharing with PU must be done in a controlled way so that the PU operation in the particular frequency band is not disturbed. Furthermore, the IEEE standard 802.22 for unlicensed access to the TV bands is in its final stages of development
[14]. Recently, Ofcom released Digital Dividend Review Statement (DDRS) which shows a radical shift in spectrum sharing policy in the UK and Ofcom is proposing to "allow license exempt use of interleaved spectrum for cognitive devices" [15]. Also, the European Commission (EU) paid much attention on dynamic spectrum management and the CR theme, and sponsored many FP5, FP6, and FP7 projects such as DRIVE [16], OverDRIVE [17], WINNER [18], E2R I/II [19], ORACLE [20], E3 [21], and "Radio Access and Spectrum" (RAS) cluster [22] tackling this issue. Similarly, several other projects outside Europe including the Defense Advanced Research Project Agency (DARPA)'s Next Generation program [23] and National Science Foundation program "NeTS-ProWiN" [24] show a significant momentum to shift spectrum access policy.

The cognition capability of a CR can make opportunistic spectrum access possible which can be implemented either by knowledge management mechanisms or by spectrum sensing functionality. A mobile network operator, for example, can equip the terminals with management mechanisms to select the most appropriate radio access technology of its heterogenous infrastructure $[25,26]$. Concentrating on spectrum sensing, observations of a single CR are not always trustworthy because a CR may have good line of sight with the primary receiver but may not be able to see the primary transmitter due to shadowing or fading, known as "hidden node" problem. Collaboration has been proposed as a solution to the problems that arise due to such uncertainties in the channel. It has been shown many times in the literature that spectrum sensing performance can be greatly improved by CSS when a number of SUs share their sensing information; fusion of this information leads to a final decision about the existence of the PU. For an overview of recent advances in CSS, the reader can refer to [9, 27-32]. Existence of a large number of cognitive users creating multiple CRN's is highly probable in the future communication systems. However, the CSS mechanisms generate a large amount of traffic overhead since each SU needs to transmit its own decision; therefore collaboration of users needs to be refined and optimised [9].

Various techniques for the optimisation of CSS in terms of fusion rule [29], number of users [33], and thresholds [34] have been proposed. It has been argued in the literature that fusion schemes strongly impact on the spectrum sensing performance including probabilities of detection and false alarm [29]. In CSS, a CR can transmit either its local observations (soft decision) or a 1-bit decision (hard decision) to a common receiver, often called fusion centre. When hard decisions are combined at the fusion centre, the $K$-outof $-N$ fusion rule is normally used [33]. In the literature, there are some studies on the optimisation of the $K$-outof $-N$ rule to minimise total decision error probability [29] and to maximise the SUs throughput [35]; however, those algorithms were designed for a specific scenario of TV bands sharing in an AWGN channel. A fusion rule based on selected information for spectrum sensing is considered in [36], in which only users that have sufficient information send their 1-bit decision to the fusion centre and the fusion centre employs best fusion rule based on the received information. 
A new fusion rule including "No decision" information from the cooperative nodes was proposed in [28].

The optimum fusion rule for combining soft decisions is Chair-Varshney rule which is based on log-likelihood ratio test [37]. Various other techniques for combining soft decisions are presented in [38]. However, most of the prior research work focuses on the case when SUs are far away from the primary transmitter and hence the same path loss or Signal-to-Noise Ratio (SNR) was assumed for all collaborating SUs $[9,29,33]$. The effects of different SNRs on detection performance are studied under AWGN channel conditions in [39]. Moreover, previous research highlighted CSS techniques which combine data from the CR nodes with equal weights and with perfect reporting channels [9, 40, 41], which is clearly not the case in realistic scenarios and might lead to misleading interpretation of results. The reporting channel for an ith user is defined as the channel between $i$ th user and the fusion centre. Performance of CSS with noisy reporting channels was considered for the case of hard decision fusion in [31].

Collaborative spectrum sensing schemes with weighted user contributions have been recently proposed in $[42,43]$. In [42] average signal power at an SU was exploited to assign weights to different collaborating cognitive nodes. In [43] a linear optimal strategy for CSS was presented and optimal weights for each SU in an AWGN channel were derived analytically. However, the shortcomings of existing literature in weighted CSS are in the fact that perfect reporting channels have been assumed instead of more realistic fading channels.

1.2. Major Contributions. In this paper, the optimisation of CSS is documented and optimum decision fusion is evaluated for hard and soft decision fusion at the fusion centre. Main contributions of this paper are summarised as follows.

(i) Hard decision fusion is attractive because of lower communication overhead over the reporting channels. In this paper, the problem of hard decision fusion at the fusion centre is addressed and answers this simple question: for optimal fusion does the fusion centre only need 1-bit decision? Different scenarios are considered with users close to the primary transmitter have the different SNR values. It is concluded that in order to achieve optimum spectrum sensing performance, the fusion centre must have SNR information for each CR and channel conditions along with their 1-bit decisions.

(ii) Maximum diversity in CSS is achieved when all collaborating users experience identical and independent fading or shadowing effects, which is not possible in reality if users are too close to each other. Multipath fading can be assumed to be independent from one user to another but shadowing is normally correlated over large distances. Thus, secondary users in close vicinity of each other make similar measurements and this limits the collaboration gains.
In this paper, correlated log-normal shadowing is considered among collaborative users and it is shown that correlated shadowing has direct impact on the optimal fusion rule at the fusion centre.

(iii) Genetic Algorithm- (GA-) based weighted collaborative spectrum sensing strategy is proposed in this paper to combat the effects of channel and enhance spectrum sensing performance. The proposed optimum spectrum sensing framework is based on a model that is realistic and also takes into account both channels, that is, channel between PU and SUs as well as the reporting channels. It is shown in this paper that imperfect reporting channel and different SU SNR values have direct impact on the performance of CSS. Secondary users transmit their soft decisions to the fusion centre and a global decision is made at the fusion centre which is based on a weighted combination of the local test statistics from individual SUs. The weight of each SU is indicative of its contribution to the final decision making. For example, if an SU has a high SNR signal and also has a good reporting channel (higher reporting channel gain), then it is assigned a larger contributing weight. The optimum CSS problem is formulated as a nonlinear optimisation problem in this paper. For a given probability of false alarm and channel conditions, optimal weights are chosen in such a way that it maximises global probability of detection at the fusion centre. With a realistic fading channel it is hard to derive an analytical expression for the optimum weights and hence a GA-based solution is proposed.

1.3. Organisation of the Paper. The remainder of this paper is organised as follows. In Section 2 the system model is briefly introduced and the use cases are defined. Section 3 discusses local spectrum sensing under channel fading conditions and its limitations. Section 4 briefly explains CSS and decision fusion techniques for both HDC and SDC, considered in this paper. Section 5 proposes a framework for optimisation of fusion rules for HDC. In order to achieve optimum spectrum sensing performance, GA is used to calculate the weights for each collaborative user in Section 6. Finally Section 7 concludes the paper.

\section{System Model for Cognitive Radio Network}

Consider a cognitive radio network, with $M$ cognitive users (indexed by $i \in\{1,2, \ldots, M\}$ ), and a fusion centre to sense a portion of the spectrum of bandwidth " $W$ " in order to detect the existence of the PU, as shown in Figure 1. Assume that each CR is equipped with an energy detector and is able to perform local spectrum sensing independently. Each CR makes its own observation based on the received signal, that is, noise only or signal plus noise. Hence, the spectrum sensing problem can be considered as a binary hypothesis 
testing problem with two possible hypothesis $\mathscr{H}_{0}$ and $\mathscr{H}_{1}$ defined as [38]

$$
x_{i}(t)= \begin{cases}n_{i}(t), & \mathscr{H}_{0}, \\ h_{i} s(t)+n_{i}(t), & \mathscr{H}_{1},\end{cases}
$$

where $s(t)$ is the PU signal and is assumed to be an identical and independent random process (i.i.d.) with zero mean and variance $\sigma_{s}^{2}$. For the $i$ th SU, the receiver noise is modelled as $n_{i}(t)$ which is also assumed to be an i.i.d. random process with zero mean and variance $\sigma_{n}^{2}$ and $h_{i}$ is the complex gain of the channel between the PU and the ith SU. Further, it is assumed that $s(t)$ and $n_{i}(t)$ are independent of each other. The power transmitted by the $\mathrm{PU}$ is received at the SU and the ratio of received power to the power of noise at the $S U$ is defined as the SNR at the SU energy detector. The received SNR at the $i$ th SU can be more precisely defined as

$$
\gamma_{i} \triangleq \frac{\mathrm{E}\left[\left|h_{i}\right|^{2}\right] \sigma_{s}^{2}}{\sigma_{n}^{2}} .
$$

System model and use cases for considered scenarios are shown in Figure 1. Two use cases are assumed in this paper. Use Case 1 refers to the case when PU transmitter is far away from the CRN and hence same SNR can be assumed for all SUs. In use Case 2, the PU is not far away from the $M$ SUs and each user has a different value of SNR depending on its distance from the PU and its channel conditions.

\section{Local Spectrum Sensing}

The performance of a given spectrum sensing scheme is fundamentally limited by the radio propagation channel. Typically, the effects of a radio channel can be divided into three main parts: path loss, small-scale fading, and large-scale fading (shadowing) [44]. Path loss effects are incorporated in the received SNR at a cognitive radio terminal. Small-scale fading causes rapid, random variations in the signal strength at the CR receiver and is modelled by Rayleigh fading in this paper. Shadowing is the slow variation of received signal power as the cognitive radio moves in and out of the shadow of large structures like mountains, buildings, and so forth. Shadowing is often modelled as a lognormal distributed random process that varies around a local mean given by the path loss and with the standard deviation $\sigma_{\mathrm{dB}}$ which depends on the environment [45].

3.1. AWGN Channel. In energy detection-based spectrum sensing, the received radio frequency energy in the considered channel or frequency band $W$ is measured over a time interval $T$ to determine whether the PU signal $s(t)$ is present. Assume that the time bandwidth product is always an integer and is denoted by $N=T W$. Test statistic $u_{i}$ calculated by an $i$ th user is given as

$$
u_{i}=\sum_{k=1}^{N}\left|x_{i}\left(\frac{k}{W}\right)\right|^{2}
$$

$u_{i}$ is compared with a predefined threshold $\lambda_{i}$ to get the local decision:

$$
\begin{gathered}
\mathscr{H}_{1} \\
u_{i} \gtreqless \lambda_{i} . \\
\mathscr{H}_{0}
\end{gathered}
$$

The binary decision is given by $D_{i} ; D_{i}=1$ when $u_{i}>\lambda_{i}$ and 0 otherwise. $u_{i}$ is the sum of squares of $N$ Gaussian random variables and it is well known that the sum of squares of Gaussian variables follows a chi-square distribution [46]. Thus $u_{i}$ follows a central chi-square distribution with $2 N$ degrees of freedom under hypothesis $\mathscr{H}_{0}$ and a noncentral chi-square distribution with $2 \mathrm{~N}$ degrees of freedom and non-centrality parameter of $2 N \gamma_{i}$ under hypothesis $\mathscr{H}_{1}$. Therefore, the probability density function (pdf) of random variable $U_{i}$ under the two hypotheses can be written as

$f_{U_{i}}(u)= \begin{cases}\frac{u^{N-1} e^{-u / 2}}{2^{N} \Gamma(N)}, & \mathcal{H}_{0}, \\ \frac{1}{2}\left(\frac{u}{2 N \gamma_{i}}\right)^{(N-1) / 2} e^{-\left(u+2 N \gamma_{i}\right) / 2} I_{N-1}\left(\sqrt{2 N u \gamma_{i}}\right), & \mathscr{H}_{1},\end{cases}$

where $\Gamma(\cdot)$ is the gamma function and $I_{N-1}(\cdot)$ is the modified Bessel function of the first kind. For an $i$ th user probability of false alarm, $\operatorname{Pr}\left(\mathscr{H}_{1} \mid \mathscr{H}_{0}\right)$, and detection, $\operatorname{Pr}\left(\mathscr{H}_{1} \mid \mathscr{H}_{1}\right)$ can be derived from (5) and is given as

$$
\begin{aligned}
& P_{f}^{i}=\operatorname{Pr}\left\{U_{i}>\lambda_{i} \mid \mathscr{H}_{0}\right\}=\frac{\Gamma\left(N, \lambda_{i} / 2\right)}{\Gamma(N)}, \\
& P_{d}^{i}=\operatorname{Pr}\left\{U_{i}>\lambda_{i} \mid \mathscr{H}_{1}\right\}=Q_{N}\left(\sqrt{2 N \gamma_{i}}, \sqrt{\lambda_{i}}\right),
\end{aligned}
$$

where $\Gamma(a, x)$ is incomplete gamma function and $Q_{N}(a, b)$ is the generalised Marcum $Q$-function. Detailed derivations of $P_{f}^{i}$ and $P_{d}^{i}$ are given in Appendices A and $\mathrm{B}$.

For the purpose of simplifying (5) an approximate model for energy detection-based spectrum sensing observations can be built. It has been shown in [47] that the approximated model converges faster and has lower approximation error when $N$ is asymptotically large. So when $N$ tends towards infinity (practically when $N \geq 10$ [46]), the chi-square distribution defined in (5) converges to a normal distribution, that is,

$$
U_{i} \sim \begin{cases}\mathcal{N}\left(N \sigma_{i}^{2}, 2 N \sigma_{i}^{4}\right), & \mathcal{H}_{0}, \\ \mathcal{N}\left(\left(N+\gamma_{i}\right) \sigma_{i}^{2}, 2\left(N+2 \gamma_{i}\right) \sigma_{i}^{4}\right), & \mathscr{H}_{1} .\end{cases}
$$

Similarly, $P_{f}^{i}$ and $P_{d}^{i}$ defined in (6) and (7) can be approximated as

$$
P_{f}^{i}=Q\left(\frac{\lambda_{i}-\mathbb{E}\left[U_{i} \mid \mathscr{H}_{0}\right]}{\sqrt{\operatorname{Var}\left[U_{i} \mid \mathscr{H}_{0}\right]}}\right)=Q\left(\frac{\lambda_{i}-N \sigma_{i}^{2}}{\sqrt{2 N} \sigma_{i}^{2}}\right)
$$




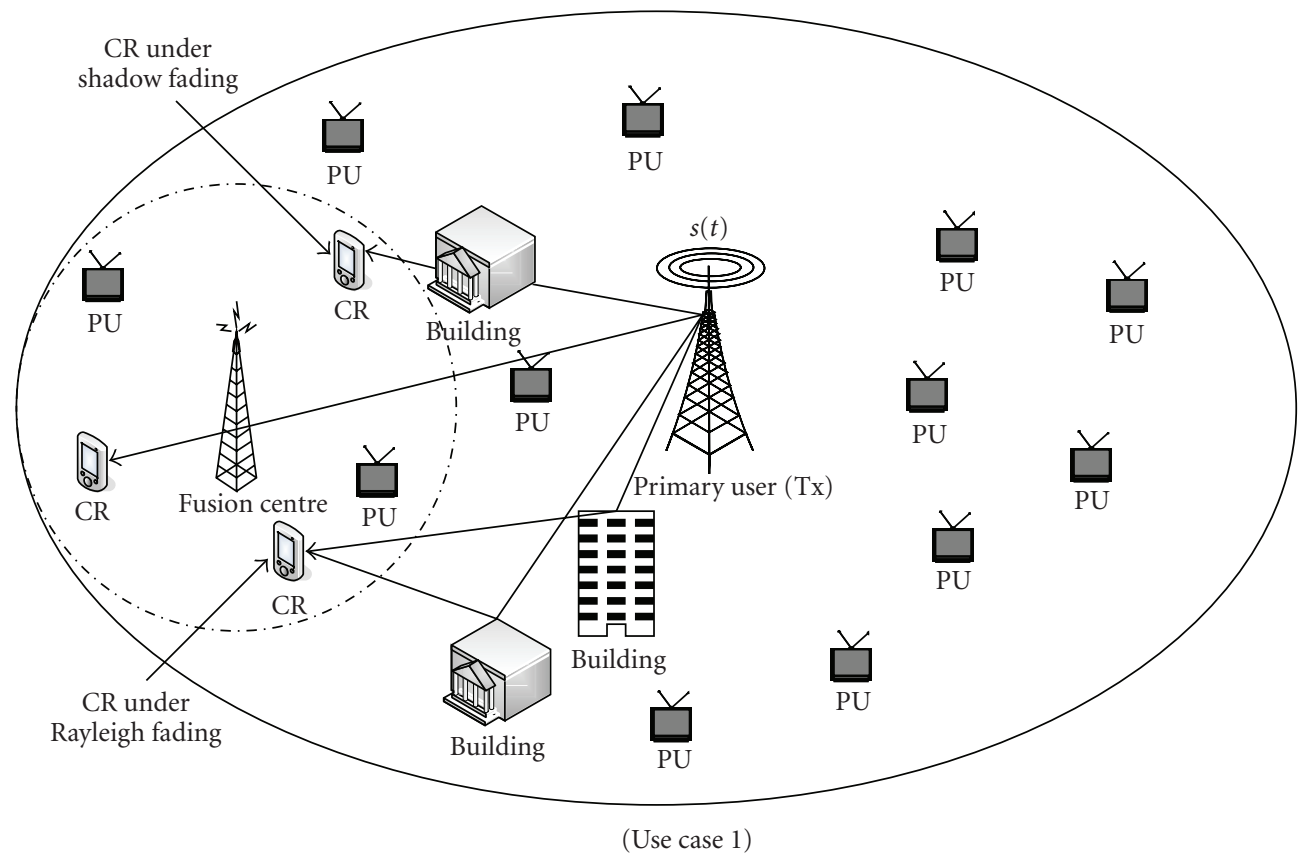

(a)

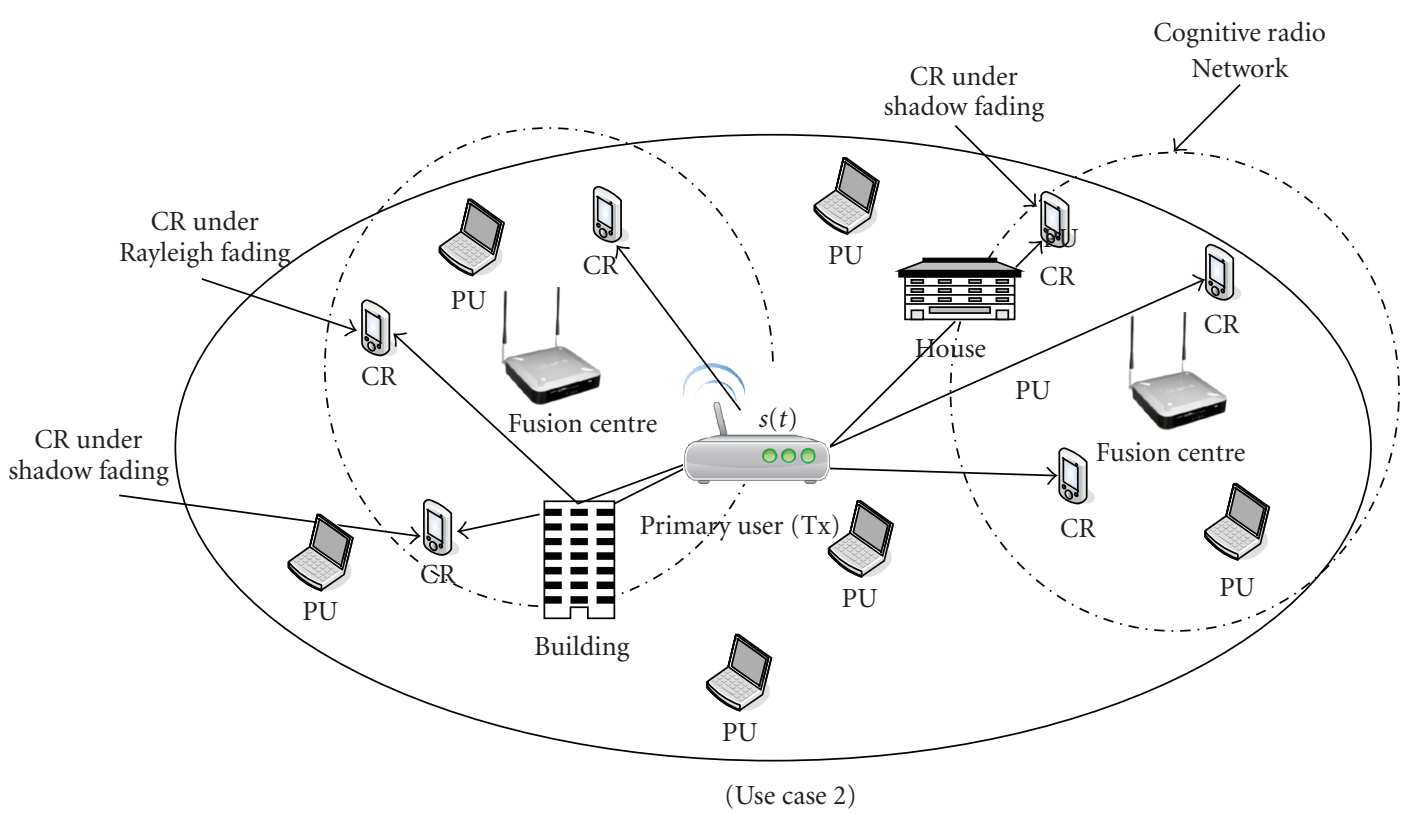

(b)

FIGURE 1: Use cases considered in paper.

$$
P_{d}^{i}=Q\left(\frac{\lambda_{i}-\mathbb{E}\left[U_{i} \mid \mathscr{H}_{0}\right]}{\sqrt{\operatorname{Var}\left[U_{i} \mid \mathscr{H}_{0}\right]}}\right)=Q\left(\frac{\lambda_{i}-\left(N+\gamma_{i}\right) \sigma_{i}^{2}}{\sqrt{2\left(N+2 \gamma_{i}\right)} \sigma_{i}^{2}}\right),
$$

where $\mathbb{E}[\cdot]$ and $\operatorname{Var} g[\cdot]$ denote expectation and variance operations, respectively.

3.2. Spectrum Sensing in Fading Channels. When the SU is in a fading channel, the channel gain $h_{i}$ for an $i$ th user is varying due to the fading and $P_{d}^{i}$ becomes conditional probability dependent on instantaneous SNR $\gamma_{i}$. As expected, $P_{f}^{i}$ is independent of $\gamma_{i}$ and remains static. Average probability of detection can be obtained by averaging instantaneous $P_{d}^{i}$ over fading statistics, where the pdf of received SNR is $f_{\gamma}(x)$ :

$$
P_{d, \mathrm{fading}}^{i}=\int_{\gamma} P_{d}^{i}(x) f_{\gamma}(x) d x
$$

When the channel is Rayleigh faded, then $\gamma_{i}$ is exponentially distributed with $\bar{\gamma}_{i}$ as its mean value. Similarly when channel is shadow faded, then $\gamma_{i}$ is log-normally distributed with 
mean $\bar{\gamma}_{i}$ and characterised by $\mathrm{dB}$-spread of shadowing $\sigma_{\mathrm{dB}}$ [38]. Hence,

$$
\begin{aligned}
& f_{\gamma_{i}}(x) \\
& = \begin{cases}\frac{1}{x} \exp \left(-\frac{x}{\bar{\gamma}_{i}}\right) ; & \text { Rayleigh Fading, } \\
\frac{\xi}{x \sigma_{\mathrm{dB}} \sqrt{(2 \pi)}} \exp \left(-\frac{10 \log _{10}(x)-\mu_{x_{\mathrm{dB}}}}{2 \sigma_{\mathrm{dB}}^{2}}\right) ; & \text { Shadow Fading, }\end{cases}
\end{aligned}
$$

where $\xi=10 / \ln (10)$ and $\mu_{x_{\mathrm{dB}}}$ is the mean of $x_{\mathrm{dB}}=10 \log (x)$. The conversion from linear mean to the log mean (in $\mathrm{dB}$ ) can be derived as [45]

$$
\mu_{x_{\mathrm{dB}}}=10 \log _{10}\left(\overline{\gamma_{i}}\right)-\frac{\sigma_{\mathrm{dB}}^{2}}{2 \xi} .
$$

Substituting (7) and (11) in (10), for Rayleigh fading channel average probability of detection for the ith user can be calculated by [38]

$$
\begin{aligned}
P_{d, \text { rayl }}^{i}= & \int_{\gamma} \frac{1}{x} Q_{N}\left(\sqrt{2 N x}, \sqrt{\lambda_{i}}\right) \exp \left(-\frac{x}{\bar{\gamma}_{i}}\right) d x \\
= & e^{-\lambda_{i} / 2} \sum_{n=0}^{N-2} \frac{1}{n !}\left(\frac{\lambda_{i}}{2}\right)^{N}+\left(\frac{1+\overline{\gamma_{i}}}{\overline{\gamma_{i}}}\right)^{N-1} \\
& \times\left[e^{-\lambda_{i} / 2\left(1+\bar{\gamma}_{i}\right)}-e^{-\lambda_{i} / 2} \sum_{n=0}^{N-2} \frac{1}{n !} \frac{\lambda_{i} \overline{\gamma_{i}}}{2\left(1+\overline{\gamma_{i}}\right)}\right] .
\end{aligned}
$$

For shadow fading, close form solution of (10) is not known and a numerical solution is required:

$$
\begin{aligned}
P_{d, \text { shadow }}^{i}= & \int_{\gamma} Q_{N}\left(\sqrt{2 N x}, \sqrt{\lambda_{i}}\right) \frac{1}{x \sigma_{\mathrm{dB}} \sqrt{2 \pi}} \\
& \times \exp \left(-\frac{10 \log (x)-\mu_{x_{\mathrm{dB}}}}{2 \sigma_{\mathrm{dB}}^{2}}\right) d x \\
= & \frac{1}{\sigma_{\mathrm{dB}} \sqrt{2 \pi}} \sum_{x=x_{0}}^{x_{f}} Q_{N}\left(\sqrt{2 N x}, \sqrt{\lambda_{i}}\right) \\
& \times \exp \left(-\frac{10 \log (x)-\mu_{x_{\mathrm{dB}}}}{2 \sigma_{\mathrm{dB}}^{2}}\right) \frac{\Delta x}{x},
\end{aligned}
$$

where $\Delta x$ and $x_{f}$ are chosen as to minimise numerical approximation error.

3.3. Numerical Evidence. The performance of local spectrum sensing is evaluated using theoretical results as well as Monte Carlo simulations by plotting complementary Receiver Operating Characteristics (ROC) curves (plot of $P_{m}=1-P_{d}$ versus $\left.P_{f}\right)$. In Monte Carlo simulations, probability of false alarm and miss detection is calculated by comparing sensing observations with a predefined threshold, and results are obtained by simulations over 1, 000, 000 noise realisations. It is assumed that $N$ is an integer value and set to be 5 .

Figure 2 shows the ROC curves for local spectrum sensing in AWGN, Rayleigh fading, and Shadowing for different values of $\sigma_{\mathrm{dB}}$. Spectrum sensing results for AWGN channel are provided for comparison and simulation results are validated by comparing with analytical results. It is clear from Figure 2 that both Rayleigh and shadow fading degrades the performance of spectrum sensing. For example, in Rayleigh fading channel, in order to achieve $P_{m}<10^{-1}$ where $P_{m}=1-P_{d}$, we need $P_{f}>0.4$ which results in poor spectrum utilisation and vice versa. Similarly, it can be seen from Figure 2 that local spectrum sensing is more difficult in shadow fading and with increase in shadowing (or $\sigma_{\mathrm{dB}}$ ) detector performance further degrades.

Another important metric to characterise spectrum sensing performance is the minimum detected SNR. This metric is defined as the lowest SNR that a sensing algorithm is able to detect with reliability of $P_{f}$ and $P_{d}$ for a given PU signal, propagation conditions and observation time. Figure 3 plots the minimum detectable SNR by a CR under different channel conditions for a targeted $P_{f}=10^{-1}$. It is clear from Figure 3 that shadowing affects detector performance more than Rayleigh fading. In order to achieve $P_{f}=10^{-1}$ in given scenario, the required SNR is around $10 \mathrm{~dB}$ while for the lower values of $\bar{\gamma}$ this is not possible as shown in Figure 3.

\section{Collaborative Spectrum Sensing}

Section 3 shows that local spectrum sensing has some limitations and it is hard to detect signals of low SNR for desired performance. Among many other challenges (e.g., see [48]) one of the most important challenges for the implementation of CRN is the hidden node problem, when a CR is shadowed or in a deep fade [41]. To address these problems multiple CRs can collaborate with each other in order to make a global decision about the existence of the PU. It has been shown by previous research that CSS can improve detection performance in the fading channels; for example, see [9] and references therein. In CSS, every SU performs its own spectrum sensing measurements and can also make a local decision on whether the PU is present or absent. All of the SUs forward their soft (local measurement) or hard (1-bit) decision to a common receiver, often called fusion centre or a band manager. Fusion centre may be centralised or distributed; in centralised CSS all the SUs send their decisions to the fusion centre, which may be an Access Point (AP) in wireless LAN or a CR base station in a cellular system, while, in distributed CSS, all the SUs may behave as a fusion centre and receive sensing information from the neighboring nodes. In both cases, fusion centre fuse collected decisions and make a final decision to declare the presence (or otherwise) of primary users in observed frequency band. The results presented in [40, 49] show that SDC outperforms HDC in terms of probability of miss detection. While HDC outperforms SDC when the 


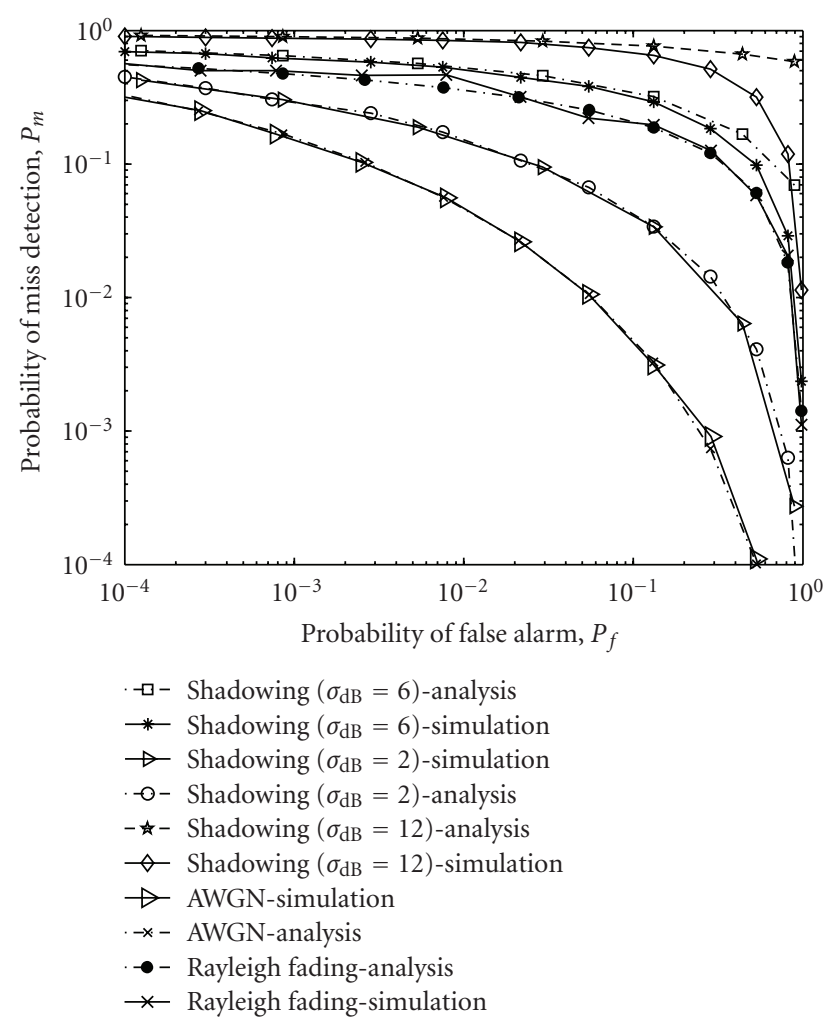

Figure 2: Receiver operating characteristics for local spectrum sensing in Rayleigh and Shadow Fading channels with $\gamma=5 \mathrm{~dB}$, $N=5$.

number of collaborative users is large [41] and further HDC needs a low-bandwidth control channel. In CSS, sharing information within CRN and combining result from various measurements is a challenging task, which is the main scope of this paper.

4.1. Hard Decision Combining. In HDC, fusion centre collects binary decisions from the individual SUs, identifies the available spectrum, and then broadcasts this information to the other SUs. The optimal decision fusion is based on Neyman-Pearson criterion by comparing Likelihood Ratio with the threshold vector as

$$
\begin{gathered}
\mathscr{H}_{1} \\
\frac{f\left(\mathbf{D} \mid \mathscr{H}_{1}\right)}{f\left(\mathbf{D} \mid \mathscr{H}_{0}\right)} \gtreqless \underline{\lambda}, \\
\mathscr{H}_{0}
\end{gathered}
$$

where $\mathbf{D}=\left[D_{1}, D_{2}, \ldots, D_{M}\right]^{T}$ denotes binary decisions from $M$ SUs and $D_{i} \in\{0,1\}, \lambda$ is the optimal threshold vector and $f\left(\mathbf{D} \mid \mathscr{H}_{0}\right)$, and $f\left(\mathbf{D} \mid \mathscr{H}_{1}\right)$ represents the probability density functions of $\mathbf{D}$ under hypothesis $\mathscr{H}_{0}$ and $\mathscr{H}_{1}$, respectively. Mathematical analysis using NeymanPearson criterion is mathematically untractable especially if the local measurements are correlated and hence sub optimal solutions are always preferable [50].

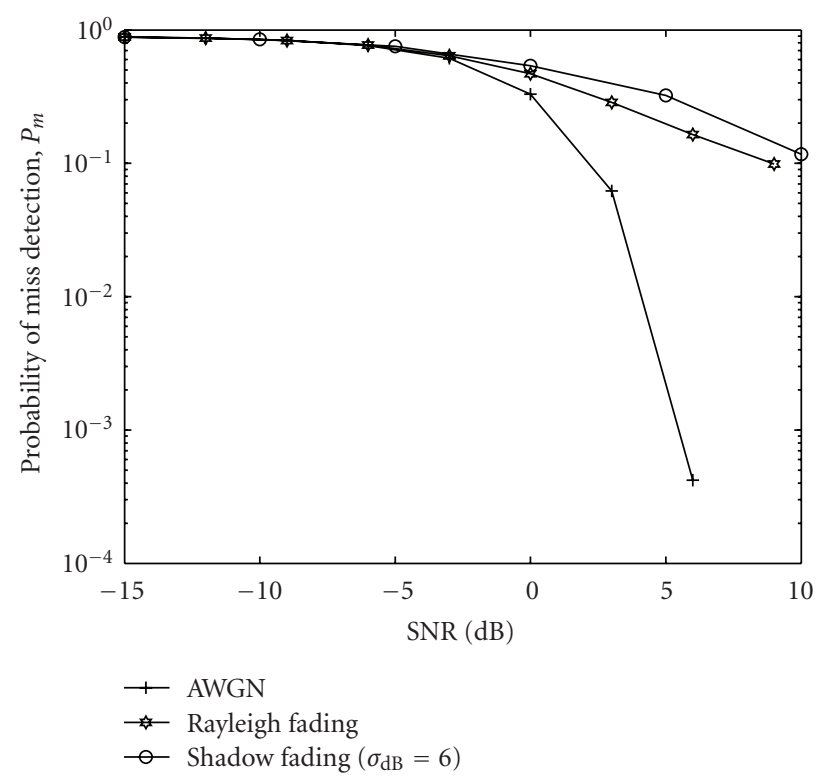

FIGURE 3: Probability of miss detection versus minimum detected SNR in shadow fading for $P_{f}=10^{-1}, \gamma_{\mathrm{dB}}=5, N=5$.

There are many other ways to combine or fuse hard decisions based on counting rules; most commonly used in the literature are $O R, A N D$ and in general $K$-out-of- $M$ fusion rule $[36,42,51]$. In $A N D$ all CRs should declare $\mathscr{H}_{1}$ in order to make a global decision that $\mathrm{PU}$ is present while in $\mathrm{OR}$ rule, fusion centre declares $\mathscr{H}_{1}$ if any of the received decision is $\mathscr{H}_{1}$. At the fusion centre, all $D_{i}$ 's are fused together according to the following fusion rule [9]:

$$
y_{c}= \begin{cases}\sum_{i=1}^{M} D_{i} \geq K, & \mathscr{H}_{1}, \\ \sum_{i=1}^{M} D_{i} \leq K, & \mathscr{H}_{0} .\end{cases}
$$

It can be seen from (16) that the $O R$ corresponds to the case when $K=1$ while for $A N D$ rule $K=M$.

It has been reported that for many cases of practical interest, the $O R$ fusion rule delivers better performance [9]. In order to demonstrate improvement in spectrum sensing performance by collaboration of SUs $O R$ fusion rule is used at the fusion centre in this section. Figures 4 and 5 show ROC curves for use Case 1 (as shown in Figure 1) with different number of CRs under i.i.d. log-normal shadowing with $\bar{\gamma}_{1}=\bar{\gamma}_{2}=\cdots=\bar{\gamma}_{i}=5 \mathrm{~dB}$ and $N=5$. In these results, AWGN curves for single users are shown for comparison. As seen in Figures 4 and 5 CSS mitigates the effects of shadow fading effectively. It can also be seen in Figure 4 that by incorporating more and more users performance even better than in the AWGN scenario can be achieved. This stems from the fact that with more number of SUs there are more chances that a single user has its instantaneous SNR above average.

As stated in Section 3 another important parameter to analyse performance of a detection algorithm is minimum 


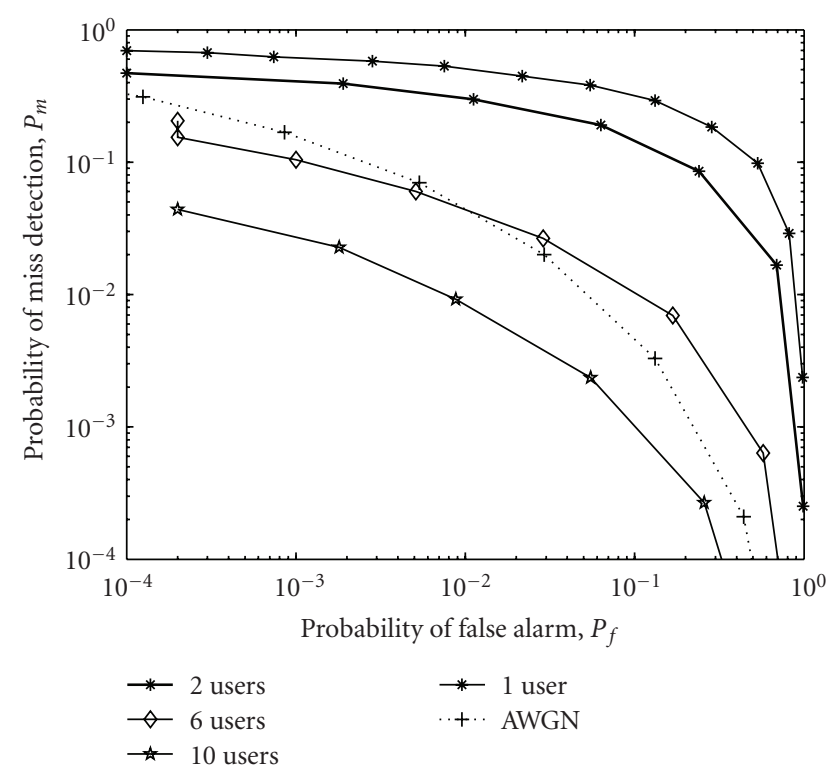

FIGURE 4: Receiver operating characteristics for collaborative spectrum sensing under shadow fading, $\sigma_{\mathrm{dB}}=6, N=5$.

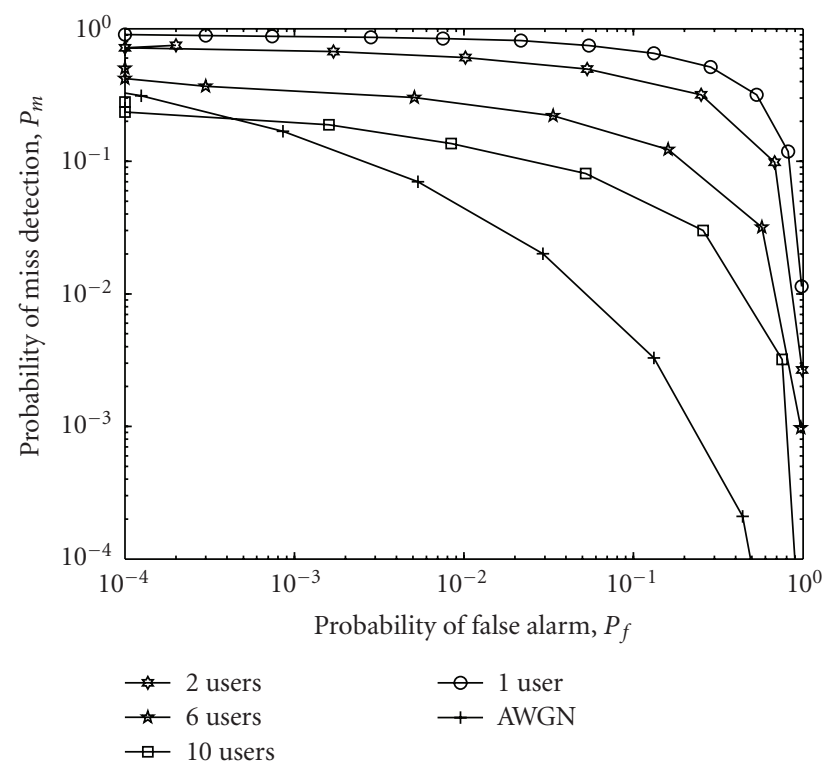

FIGURE 5: Receiver operating characteristics for collaborative spectrum sensing under shadowing, $\sigma_{\mathrm{dB}}=10, N=5$.

detected SNR. A good detection scheme must be able to detect signals of low SNR, and in Section 3 it has been shown that shadowing affects detected SNR by a user. Figure 6 shows that by incorporating a large number of users it is possible to achieve the desired performance even at low SNR levels. By comparing Figures 3 and 6 it can be seen that under shadow fading $\left(\sigma_{\mathrm{dB}}=6\right)$ and for desired performance, for example, $P_{f}=10^{-1}$ and $P_{d}=10^{-1}$ local sensing requires received signal of at least $10 \mathrm{~dB}$ while collaboration of 20 users can detect signal of SNR as low as $-15 \mathrm{~dB}$.

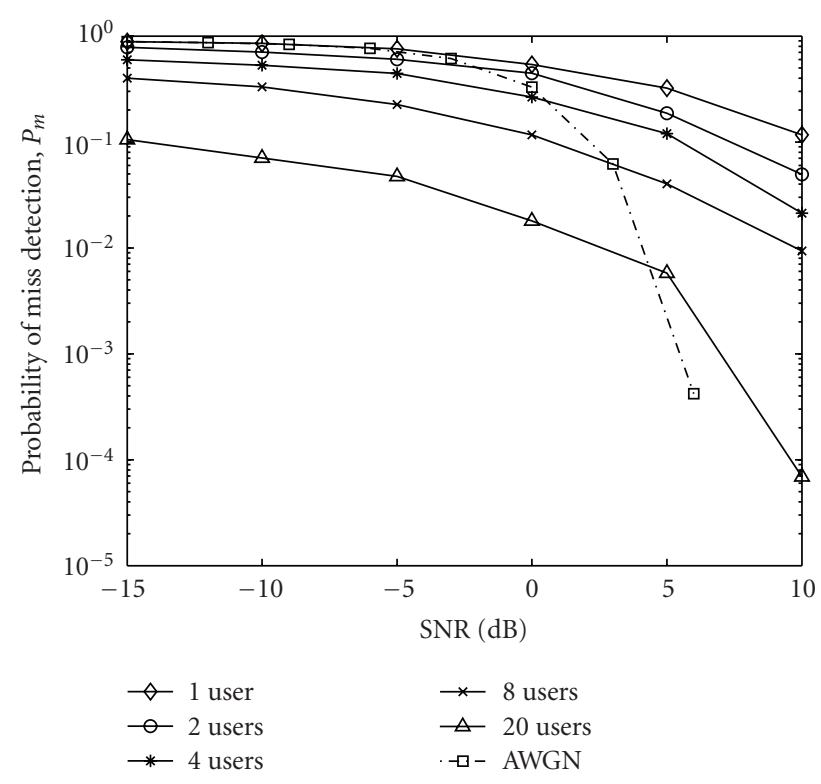

Figure 6: Probability of miss detection versus minimum detected SNR in shadow fading, $P_{f}=10^{-1}, \sigma_{\mathrm{dB}}=6, \gamma=5 \mathrm{~dB}, N=5$.

4.2. Soft Decision Combining. In order to simplify the analysis with fusion of soft decisions, it has been assumed that the value of $N$ is large. With this assumption the summary statistics at local secondary nodes $\mathbf{U}$ (as defined in (8)) can be considered, which is transmitted to the fusion centre through the reporting channels. In this paper realistic noisy reporting channels with variable channel gains are considered. A system model is shown in Figure 13.

4.2.1. Equal Gain Combining. Statistics of local observations for an $i$ th SU after passing through the channel of gain $g_{i}$ and noise $n_{i} \sim \mathcal{N}\left(0, \delta_{i}^{2}\right)$ is

$$
y_{i} \sim \begin{cases}\mathcal{N}\left(N g_{i} \sigma_{i}^{2}, 2 N g_{i}^{2} \sigma_{i}^{4}+\delta_{i}^{2}\right), & \mathscr{H}_{0}, \\ \mathcal{N}\left(\left(N+\bar{\gamma}_{i}\right) g_{i} \sigma_{i}^{2}, 2\left(N+2 \bar{\gamma}_{i}\right) g_{i}^{2} \sigma_{i}^{4}+\delta_{i}^{2}\right), & \mathscr{H}_{1},\end{cases}
$$

where $\delta_{i}^{2}$ is the noise variance of the $i$ th reporting channel. For the soft decision fusion scheme, fusion centre decides between $\mathscr{H}_{0}$ and $\mathscr{H}_{1}$ by comparing sum of individual observations $y_{c}$ with a global threshold $\lambda_{c}$ :

$$
\begin{gathered}
\mathscr{H}_{1} \\
y_{c}=\sum_{i=1}^{M} y_{i} \gtreqless \lambda_{c} . \\
\mathscr{H}_{0}
\end{gathered}
$$

4.2.2. Weighted Combining. In weighted combining, global test statistics is calculated at the fusion centre by assigning weights $w_{i}$ to the received observation from an ith user $y_{i}$ by

$$
y_{c}=\sum_{i=1}^{M} w_{i} \cdot y_{i}=\mathbf{w}^{T} \mathbf{y},
$$



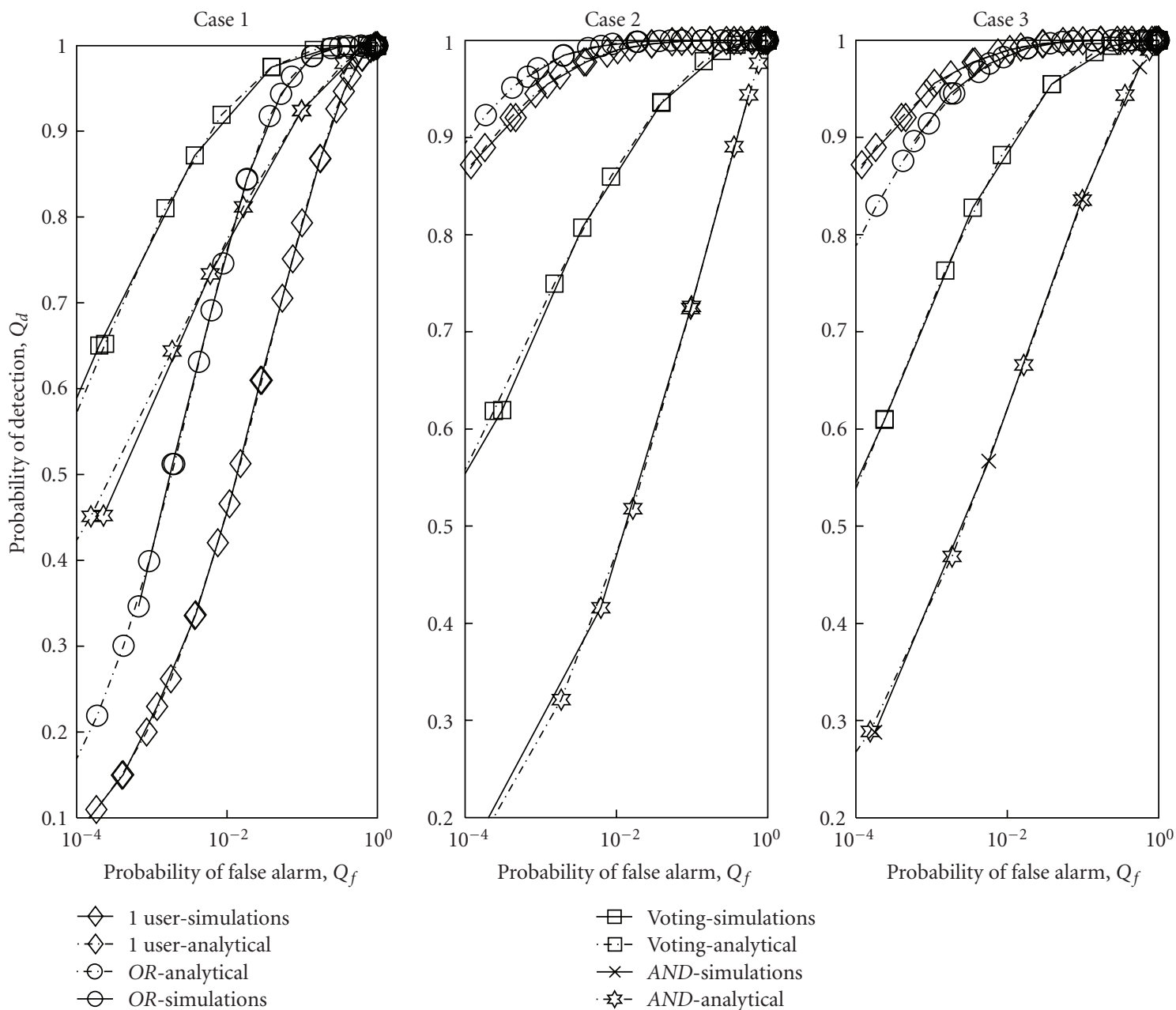

FIGURE 7: Receiver operating characteristics for 5 collaborating users in AWGN Channel in 3 cases: Case 1 (all users have similar SNR), Case 2 (half of the users have high SNR), and Case 3 (only one user has high SNR).

where $\mathbf{w}=\left[w_{1}, w_{2}, \ldots, w_{M}\right]^{T} \in \mathbb{R}^{M \times 1}$ and the received decision vector at the fusion centre is defined as $\mathbf{y}=$ $\left[y_{1}, y_{2}, \ldots, y_{M}\right]^{T} \in \mathbb{R}^{M \times 1}$. Weight vector $\mathbf{w}$ at the fusion centre satisfies $\sum_{i=1}^{M} w_{i}=1$. From (17) and (19) the distribution of $y_{c}$ is given as

$$
\sim \begin{cases}y_{c} & \begin{cases}\mathcal{N}\left(\sum_{i=1}^{M} N g_{i} \sigma_{i}^{2} w_{i}, \sum_{i=1}^{M}\left(2 N g_{i}^{2} \sigma_{i}^{4} w_{i}^{2}+\delta_{i}^{2} w_{i}^{2}\right)\right) ; & \mathscr{H}_{0} \\ \mathcal{N}\left(\sum_{i=1}^{M}\left(\left(N+\bar{\gamma}_{i}\right) g_{i} \sigma_{i}^{2} w_{i}\right),\right. \\ \left.\sum_{i=1}^{M}\left(2\left(N+2 \bar{\gamma}_{i}\right) g_{i}^{2} \sigma_{i}^{4} w_{i}^{2}+\delta_{i}^{2} w_{i}^{2}\right)\right) ; & \mathscr{H}_{1} .\end{cases} \end{cases}
$$

Assume $\mathbf{h}=\left[h_{1}, h_{2}, \ldots, h_{M}\right]^{T} \in \mathbb{R}^{M \times 1}, \mathbf{g}=\left[g_{1}, g_{2}, \ldots\right.$, $\left.g_{M}\right]^{T} \in \mathbb{R}^{M \times 1}, \boldsymbol{\gamma}=\left[\gamma_{1}, \gamma_{2}, \ldots, \gamma_{M}\right]^{T} \in \mathbb{R}^{M \times 1}, \boldsymbol{\sigma}=$ $\left[\sigma_{1}^{2}, \sigma_{2}^{2}, \ldots, \sigma_{M}^{2}\right]^{T} \in \mathbb{R}^{M \times 1}$, and $\boldsymbol{\delta}=\left[\delta_{1}^{2}, \delta_{2}^{2}, \ldots, \delta_{M}^{2}\right]^{T} \in$ $\mathbb{R}^{M \times 1}$. Furthermore, defined matrices $\Sigma, \Delta, \Gamma$, and $\mathbf{G}$ that all belong to $\mathbb{R}^{M \times M}$ represent the diagonal matrices formed by placing the vectors $\boldsymbol{\sigma}, \boldsymbol{\delta}, \bar{\gamma}$, and $\mathbf{g}$ on the diagonal, respectively. The statistics of $y_{c}$ under $\mathscr{H}_{0}$ and $\mathscr{H}_{1}$ can be written as

$$
\begin{aligned}
\mathrm{E}\left[y_{c} \mid \mathscr{H}_{0}\right] & =N \mathbf{g}^{T} \mathbf{\Sigma} \mathbf{w}, \\
\operatorname{Var}\left[y_{c} \mid \mathscr{H}_{0}\right] & =\mathbf{w}^{T}\left[2 N \mathbf{G}^{2} \boldsymbol{\Sigma}^{2}+\Delta\right] \mathbf{w}, \\
\mathrm{E}\left[y_{c} \mid \mathscr{H}_{1}\right] & =\mathbf{g}^{T}((N \mathbf{I}+\boldsymbol{\Gamma}) \odot \boldsymbol{\sigma}) \mathbf{w}, \\
\operatorname{Var}\left[y_{c} \mid \mathscr{H}_{1}\right] & =\mathbf{w}^{T}\left[2(N \mathbf{I}+2 \boldsymbol{\Gamma}) \mathbf{G}^{2} \boldsymbol{\Sigma}^{2}+\Delta\right] \mathbf{w} .
\end{aligned}
$$

To make a decision on the presence of a primary transmitter, the global decision statistic $y_{c}$ as defined in (21) is compared with a threshold $\lambda_{c}$. Global probability of false 

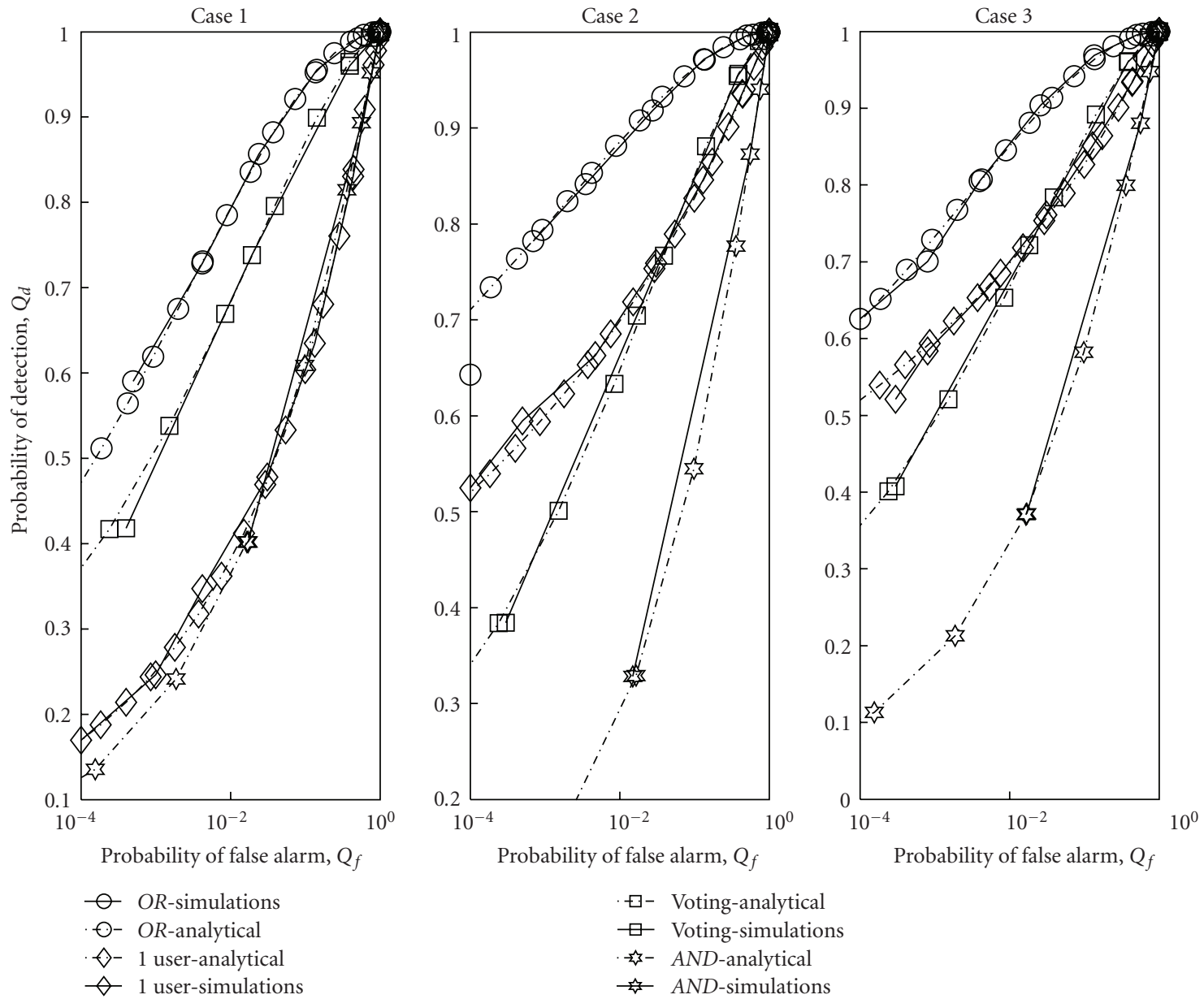

FIgURE 8: Receiver operating characteristics for 5 collaborating users in Rayleigh fading Channel in 3 cases: Case 1 (all users have similar SNR), Case 2 (half of the users have high SNR), and Case 3 (only one user has high SNR).

alarm and detection at the fusion centre, as denoted by $Q_{f}$ and $Q_{d}$, are given as

$$
\begin{aligned}
& Q_{f}=Q\left(\frac{\lambda_{c}-N \mathbf{g}^{T} \mathbf{\Sigma} \mathbf{w}}{\sqrt{\mathbf{w}^{T}\left[2 N \mathbf{G}^{2} \boldsymbol{\Sigma}^{2}+\Delta\right] \mathbf{w}}}\right), \\
& Q_{d}=Q\left(\frac{\lambda_{c}-\mathbf{g}^{T}((N \mathbf{I}+\boldsymbol{\Gamma}) \odot \boldsymbol{\sigma}) \mathbf{w}}{\sqrt{\mathbf{w}^{T}\left[2(N \mathbf{I}+2 \boldsymbol{\Gamma}) \mathbf{G}^{2} \boldsymbol{\Sigma}^{2}+\Delta\right] \mathbf{w}}}\right),
\end{aligned}
$$

where $Q(\cdot)$ is the tail probability of the normalised Gaussian distribution.

\section{Optimised User Collaboration Scheme for HDC}

Section 4.1 shows that collaboration of SUs improves spectrum sensing performance by utilising space diversity of users. In this section, the problem of hard decision fusion at the fusion centre is considered in the presence of i.i.d. and spatially correlated shadowing. In the past, emphasis was given to collaborative spectrum sensing when all users have same received SNR; however, in this section, a scenario where users have different $\gamma_{i}$ with AWGN and log-normal shadowing is considered. Three different cases in use Case 2 are considered here which represents three different scenarios depending on the location of PU and SUs. Case 1 refers to a scenario in which all the SUs are relatively close to each other and hence having similar values of SNR. Case 2 depicts the situation when half of the collaborating users have high SNR values while in Case 3 only one use has a high SNR value as compared to other collaborating SUs.

Different decision fusion schemes at the fusion centre including $O R, A N D$, Voting, and 1-user cases are considered. In Voting-based decision fusion scheme all SUs vote and fusion centre declare an opportunity if the majority of the collaborative SUs declare an opportunity. In 1-user case although fusion centre receives information from all users, it uses only one user information in order to make a global decision.

\subsection{Independent and Identically Distributed Shadowing}

5.1.1. Mathematical Formulation. The global probability of detection $Q_{d}$ and probability of false alarm $Q_{f}$ at the fusion 

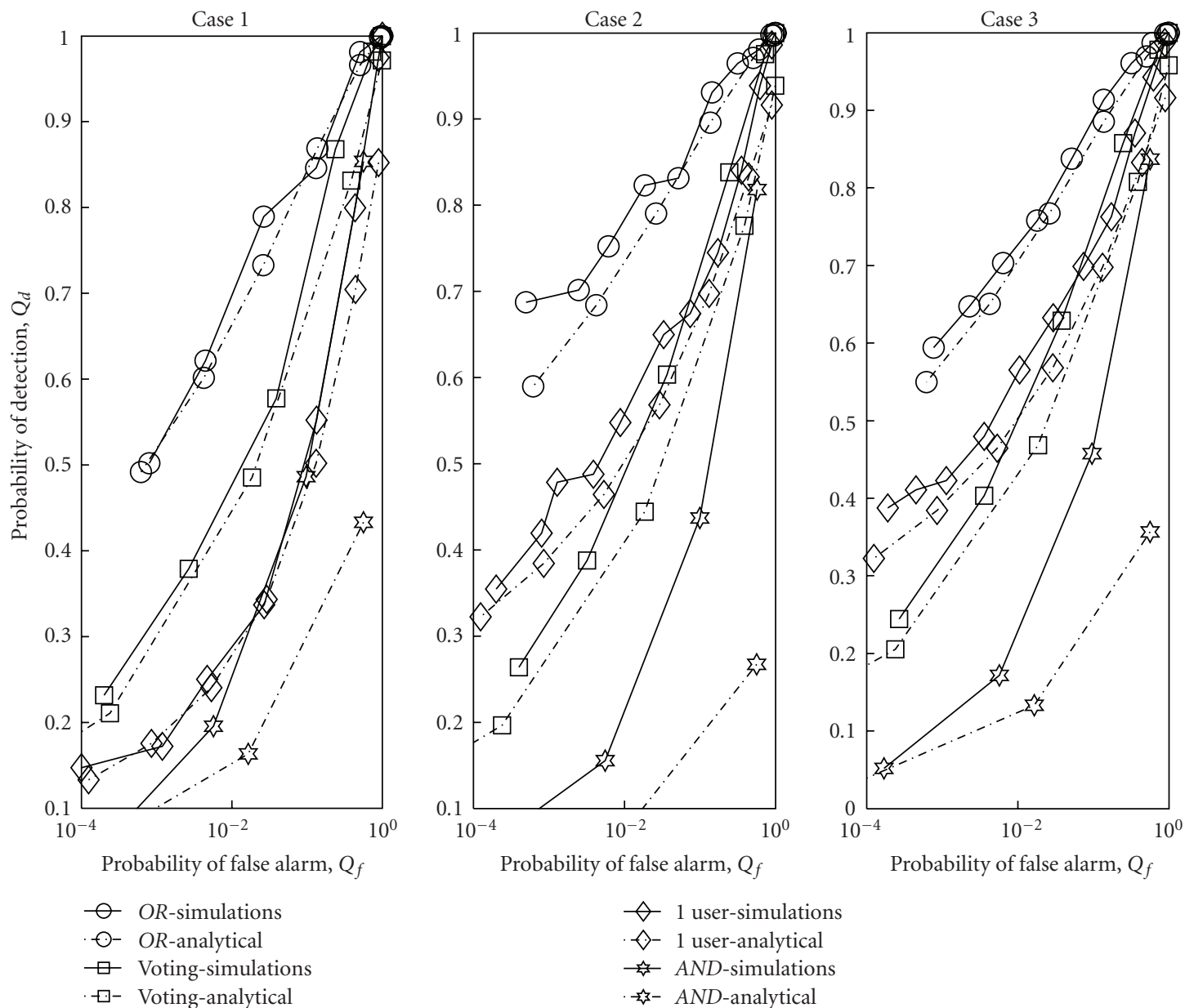

FIGURE 9: Receiver operating characteristics for 5 collaborating users in shadow fading $\left(\sigma_{\mathrm{dB}}=6\right)$ in 3 cases: Case 1 (all users have similar SNR), Case 2 (half of the users have high SNR), and Case 3 (only one user has high SNR).

centre can be expressed as a function of the probability of detection (or false alarm) of each SU, obtaining the joint probability of $M$ independent events as

$$
\begin{aligned}
& Q_{d}=R(\mathbf{D}) \prod_{S_{0}}\left(1-P_{d}^{i}\right) \prod_{S_{1}} P_{d}^{i}, \\
& Q_{f}=R(\mathbf{D}) \prod_{S_{0}}\left(1-P_{f}^{i}\right) \prod_{S_{1}} P_{f}^{i} .
\end{aligned}
$$

$S$ represents the set of all secondary users with $\delta=\varsigma_{0} \cup \wp_{1}$ where $\delta_{0}$ is the group of SUs that has decided that PU signal is absent while $\delta_{1}$ is the group of SUs that has decided that $\mathrm{PU}$ signal is absent and $R(\mathbf{D})$ is the decision fusion rule at the fusion centre. Value of $R(\mathbf{D})$ depends on what type of fusion rule is used at the fusion centre. So for the given formulation, $K$-out-of- $M$ rule can be formulated as

$$
R(\mathbf{D})= \begin{cases}1 \text { (PU present) } & \text { if } \sum_{i=1}^{M} D_{i} \geq K, \\ 0 \text { (PU absent) } & \text { if } \sum_{i=1}^{M} D_{i}<K\end{cases}
$$

For the fusion rules considered in this section, $K$ is given as

$$
K= \begin{cases}1, & \text { OR, } \\ M, & \text { AND, } \\ \left\lceil\frac{M}{2}\right\rceil, & \text { Voting }\end{cases}
$$

where $\lceil\cdot\rceil$ is the ceil function.

For 1-user rule,

$$
R(\mathbf{D})= \begin{cases}1, & \text { if } D_{i}=1, \\ 0, & \text { otherwise, }\end{cases}
$$

where the $i$ th user is chosen as

$$
i=\arg \max _{j}\left\{\gamma_{j}\right\} .
$$

5.1.2. Simulation Results. Figure 7 shows collaborative spectrum sensing performance when 5 SUs collaborate with each other and make collaborative decision; analytical results validating the simulation results are shown. When all users 
have similar $\gamma_{i}$ (Case 1) in AWGN channel, then the optimal decision fusion rule is Voting rule as evident from Figure 7. When half of the users have high $\gamma_{i}$ and half of the users have low $\gamma_{i}$ (Case 2), then the optimal decision rule is OR. Case 3 refers to the situation when only one user has higher $\gamma_{i}$ than others; in this case the collaborative spectrum sensing works even worse than a single node. From these results it can be concluded that it is not necessary that collaboration of users always improves spectrum sensing performance and in order to derive an optimum performance individual $\gamma_{i}$ should be considered. Without knowing $\gamma_{i}$ the performance is always suboptimal; so in the proposed scenario it is suggested that users estimate local $\gamma_{i}$ and send this information along with their 1-bit decision. Local SNR can be estimated by using a test statistics defined in (5) as

$$
\hat{\gamma}_{i}=\frac{1}{Z} \sum_{i=1}^{Z} u_{i}-X
$$

where $\hat{\gamma}$ is estimated SNR, $Z$ is the number of test statistics, and $X$ is $\mathrm{E}\left(u_{i} \mid \mathscr{H}_{0}\right)$.

In Rayleigh fading and shadowing, collaborative spectrum sensing is an ideal solution because diversity gain achieved by collaboration effectively cancels the deleterious effects of fading. Figures 8 and 9 show detection performance under Rayleigh fading and shadowing with the three cases considered in this section. Value of dB-spread is assumed to be $6 \mathrm{~dB}$ for the shadowing while other parameters remain the same. As can be seen from these results, in all three cases spectrum sensing performance is superior if $O R$ fusion rule is used at the fusion centre. So it can be concluded from simulation results that under Rayleigh fading and shadowing with i.i.d. measurements most optimal fusion rule is $O R$ rule and collaboration of users is required. Further, with the increase of shadowing, sensing performance of two user collaboration with highest $\gamma_{i}$ is better than collaboration of all users. It can be concluded that even in fading or shadowing it is important for the fusion centre to know the SNR values of the users to make a decision about which fusion rule gives better performance.

5.2. Spatially Correlated Shadowing. Up to this point, it is assumed that all collaborating cognitive users have identical and independent shadowing. However, usually there is a degree of spatial correlation associated with log-normal shadowing [52] and assumption of identically and independent (i.i.d.) shadowing is not always true. In this section, the impact of spatially correlated shadowing on decision fusion when users have different SNR is studied under different channel conditions. It is concluded that correlation has a direct impact on the optimum decision fusion rule at the fusion centre.

It is logical to think that spatially correlated shadowing would degrade the performance of CSS because such users are likely to experience similar observations thereby countering collaborative gains. In this paper correlated shadowing is modelled using the exponential correlation model [52]:

$$
r(d)=e^{-a d}
$$

where $r(d)$ is the correlation matrix, $d$ is the distance between two secondary users, and $a$ is a constant depending on the environment. Based on measurements reported in [52], $a \approx 0.12 / \mathrm{m}$ for urban environment and $a \approx 0.002 / \mathrm{m}$ for suburban environment.

5.2.1. Mathematical Formulation. Assume that $\gamma_{i}$ is the received SNR at the $i$ th $\mathrm{SU}$ on a logarithmic scale. Hence under shadow fading $\gamma_{i}$ has a Gaussian distribution with variance of $\sigma_{\mathrm{dB}}^{2}$ and a mean value of $\mu_{\gamma}$ (in $\left.\mathrm{dB}\right)$. The value of $\mu_{\gamma}$ is determined by the distance dependent path loss. Under two hypotheses $\mathscr{H}_{0}$ and $\mathscr{H}_{1}$ the distribution of $\gamma_{i}$ for $M$ SUs under spatially correlated shadowing can be expressed as

$$
\gamma_{\mathrm{dB}} \sim \begin{cases}\mathcal{N}\left(0 \times \mathbf{u}_{M}, \sigma_{\mathrm{dB}}^{2} \boldsymbol{\Xi}\right), & \mathscr{H}_{0}, \\ \mathcal{N}\left(\boldsymbol{\mu}_{\gamma}, \sigma_{\mathrm{dB}}^{2} \boldsymbol{\Xi}\right), & \mathscr{H}_{1},\end{cases}
$$

where $\gamma_{\mathrm{dB}}=\left[\gamma_{1}, \gamma_{2}, \ldots, \gamma_{M}\right]^{T}, \mathbf{u}_{M}$ is an $M \times 1$ vector of all ones, and $\boldsymbol{\Xi}$ is the normalised covariance matrix of $\gamma_{\mathrm{dB}}$. Using the exponential correlation model defined in (29), the covariance matrix $\Xi$ is an $M \times M$ matrix. Assuming that all SUs are uniformly distributed in a 1-dimensional plane within a total distance of $\kappa$, the elements of covariance matrix are given as

$$
\boldsymbol{\Xi}_{i, j}=e^{(-a \kappa /(M-1))|i-j|} .
$$

Hence, the covariance matrix $\boldsymbol{\Xi}$ can be expressed as

$$
\Xi=\left[\begin{array}{ccccc}
1 & \mathcal{A} & \mathcal{B} & \cdots & e^{-a \kappa} \\
\mathcal{A} & 1 & \mathcal{A} & \cdots & e^{-a \kappa|M-2| /(M-1)} \\
\vdots & \vdots & \vdots & \cdots & \vdots \\
e^{-a \kappa} & \mathcal{B} & e^{-3 a \kappa /(M-1)} & \cdots & 1
\end{array}\right]
$$

where $\mathcal{A}$ denotes $e^{-a \kappa /(M-1)}$ and $\mathcal{B}$ denotes $e^{-2 a \kappa /(M-1)}$.

The probability density function of $\gamma_{i}$ can be expressed for the $M$ collaborative SUs having correlated shadow fading as

$$
f\left(\gamma_{\mathrm{dB}}\right)=\frac{1}{\sqrt{2 \pi} \sigma_{\mathrm{dB}}^{2}} \boldsymbol{\Xi}^{-1} \exp \left\{-\frac{\left(\gamma_{\mathrm{dB}}-\mu_{\gamma}\right)^{2}}{2 \sigma_{\mathrm{dB}}^{2}} \boldsymbol{\Xi}^{-1}\right\} .
$$

From (32) it is clear that $\Xi$ is a diagonal constant matrix or Toeplitz matrix, and its inverse may be expressed as [53]

$$
\Xi^{-1}=\frac{1}{1-e^{2 a \kappa /(M-1)}}\left[\begin{array}{ccccc}
1 & -\mathcal{A} & 0 & \cdots & 0 \\
-\mathcal{A} & 1+e^{-2 a \kappa /(M-1)} & -\mathcal{A} & \cdots & 0 \\
\vdots & \vdots & \vdots & \cdots & \vdots \\
0 & 0 & \cdots & -\mathcal{A} & 1
\end{array}\right] \text {, }
$$

where $\mathcal{A}$ denotes $e^{-a \kappa /(M-1)}$.

5.2.2. Simulation Results. It is shown in this section that spatial correlation among users directly impacts the decision fusion at the fusion centre. Figures 10, 11, and 12 show ROC 


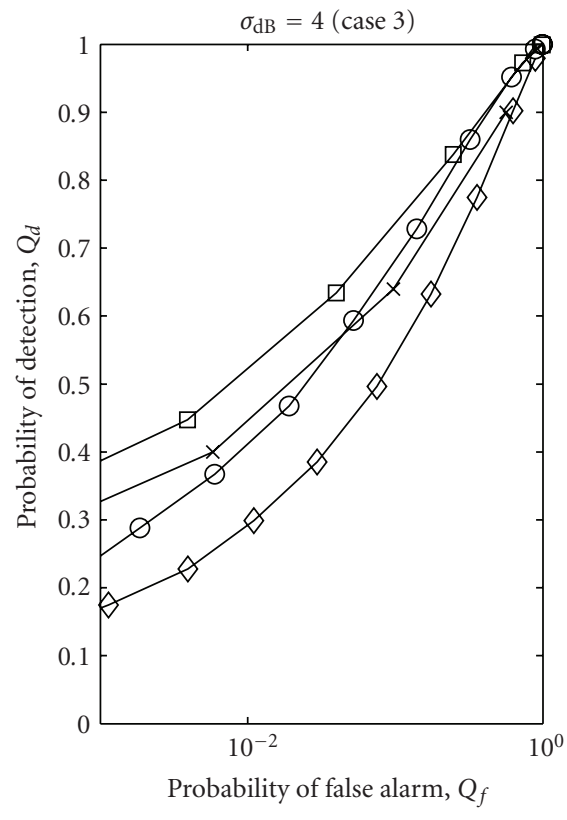

(a)

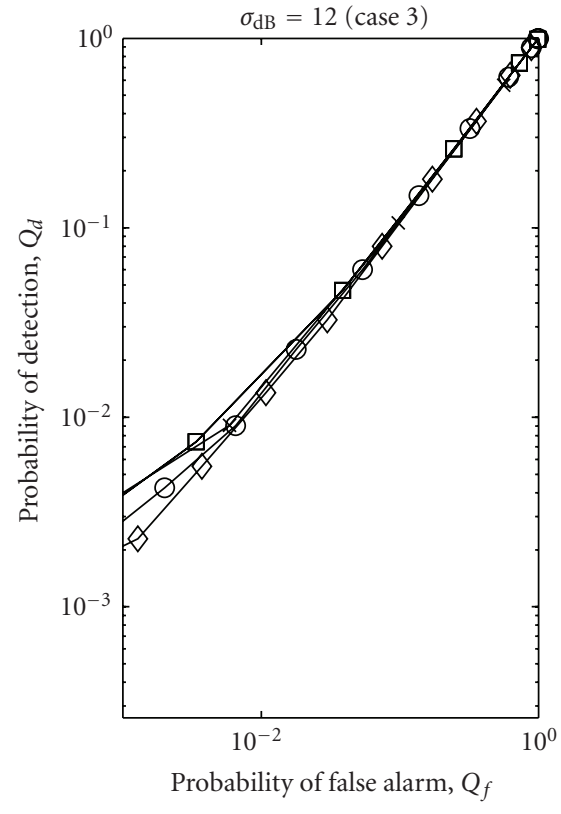

(b)

FIGURE 10: Receiver operating characteristics for 5 collaborating users in spatially correlated shadowing fading (Case 1: all users have similar SNR).

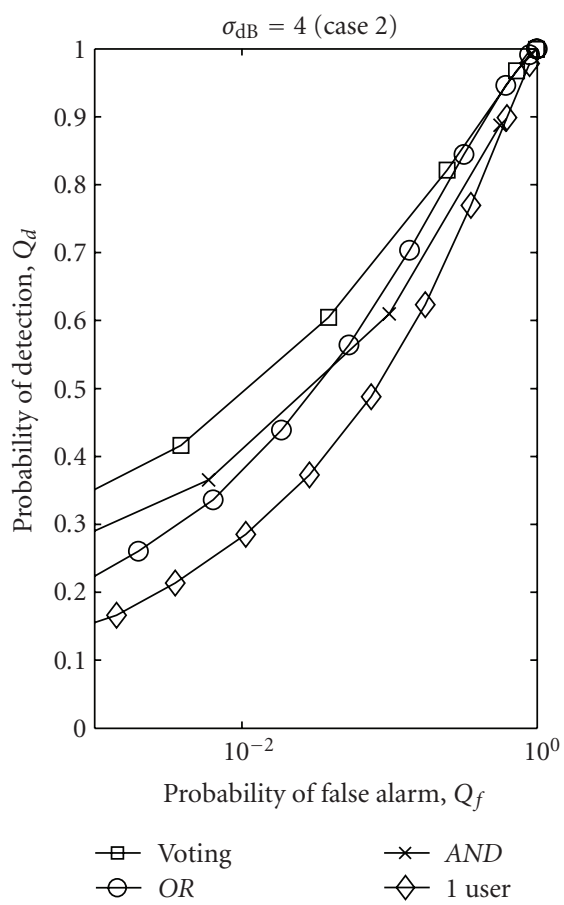

(a)

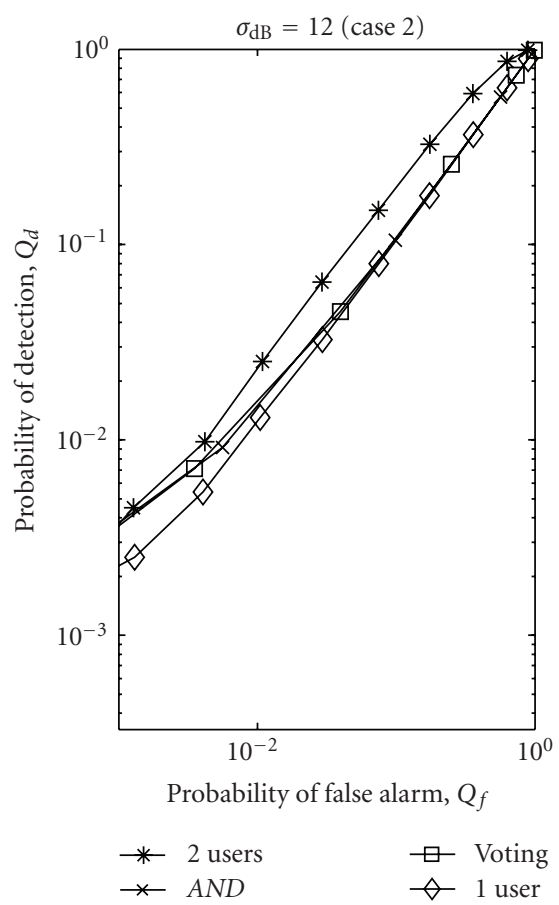

(b)

FIGURE 11: Receiver operating characteristics for 5 collaborating users in spatially correlated shadowing fading (Case 2: half of the users have high SNR).

curves of 5 collaborating users under spatially correlated shadowing with $\mathrm{dB}$-spread of $4 \mathrm{~dB}$ and $12 \mathrm{~dB}$ for the three cases defined in Section 5.1. In case of correlated shadowing with lower values of $\sigma_{\mathrm{dB}}$, the Voting fusion rule outperforms $O R$ fusion rule and performance of $A N D$ fusion rule is better than $O R$. This is due to the fact that all secondary users are close to each other and have similar values of $\gamma_{i}$; hence user observations are similar to each other. However, sensing performance in heavily shadowed environment (e.g., when $\sigma_{\mathrm{dB}}=12$ ) for all fusion schemes is almost similar in all 


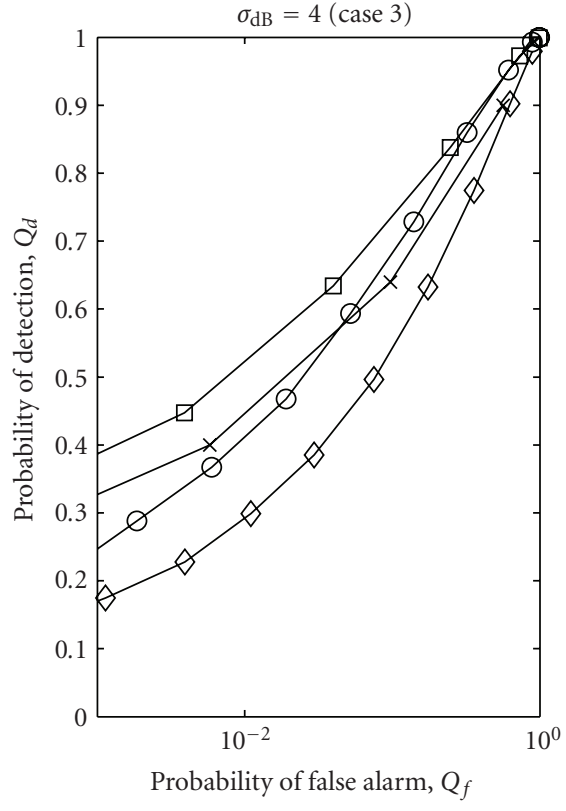

(a)

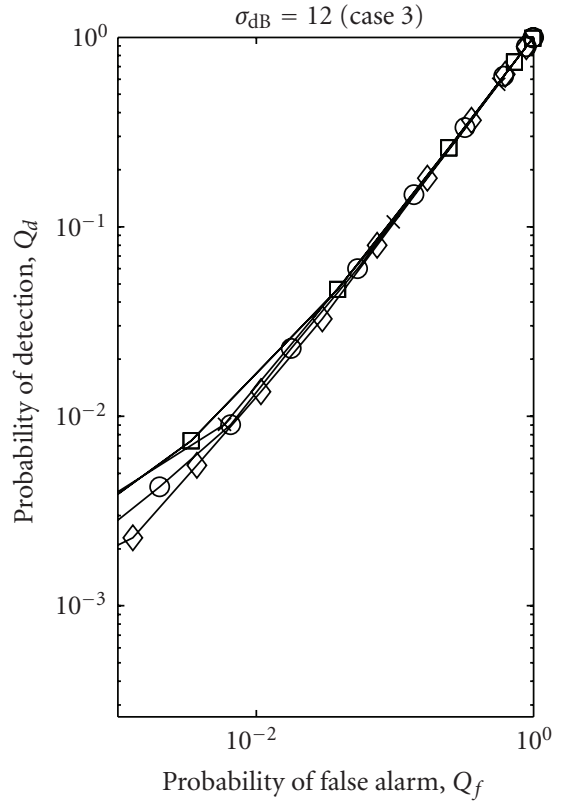

(b)

FIGURE 12: Receiver operating characteristics for 5 collaborating users in spatially correlated shadowing fading (Case 3: only one user has high SNR).

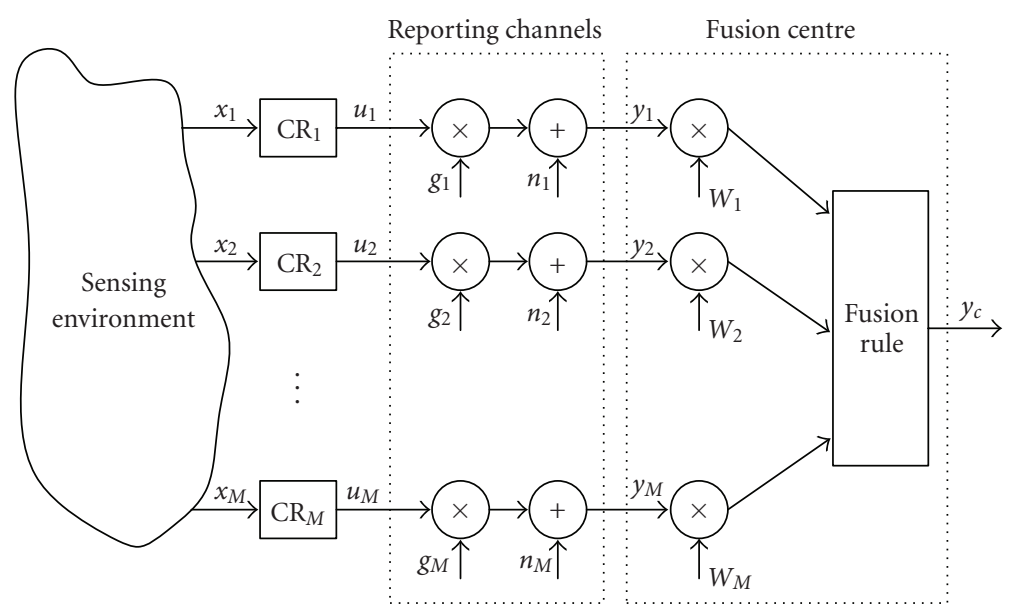

FIGURE 13: Schematic diagram of weighted collaboration at fusion centre for soft decision combining with imperfect reporting channels.

three cases. Hence, it can be seen from Figures 10, 11, and 12 that for optimal decision fusion at the fusion centre, it is important to consider the effects and degree of correlation among users.

\section{Optimised User Collaboration Scheme for SDC}

In this section, goal is to optimise CSS when collaborating SUs send their soft decisions to the fusion centre by maximising the global probability of detection (or alternatively minimising global probability of miss detection) for a given value of probability of false alarm and channel conditions.
Referring to Section 4.2 for the framework of soft decision combing at the fusion centre, global probability of detection can be written in terms of global probability of false alarm (using (22)):

$$
\begin{array}{r}
Q_{d}=Q\left(\frac{\sqrt{\mathbf{w}^{T}\left[2 N \mathbf{G}^{2} \boldsymbol{\Sigma}^{2}+\Delta\right] \mathbf{w}} Q^{-1}\left(Q_{f}\right)}{\sqrt{\mathbf{w}^{T}\left[2(N \mathbf{I}+2 \boldsymbol{\Gamma}) \mathbf{G}^{2} \boldsymbol{\Sigma}^{2}+\Delta\right] \mathbf{w}}}\right. \\
\left.+\frac{N \mathbf{g}^{T} \boldsymbol{\Sigma} \mathbf{w}-\mathbf{g}^{T}((N \mathbf{I}+\boldsymbol{\Gamma}) \odot \boldsymbol{\sigma}) \mathbf{w}}{\sqrt{\mathbf{w}^{T}\left[2(N \mathbf{I}+2 \boldsymbol{\Gamma}) \mathbf{G}^{2} \boldsymbol{\Sigma}^{2}+\Delta\right] \mathbf{w}}}\right) .
\end{array}
$$




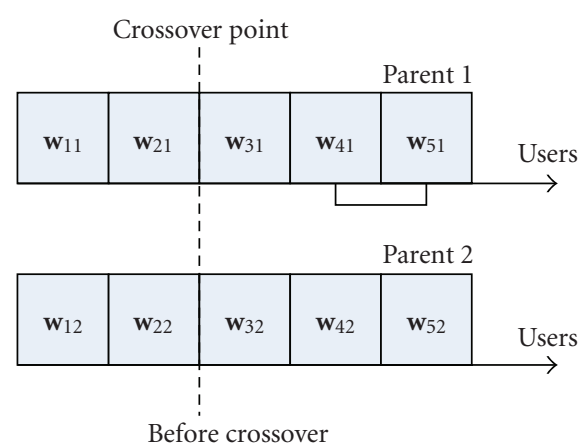

Child 1
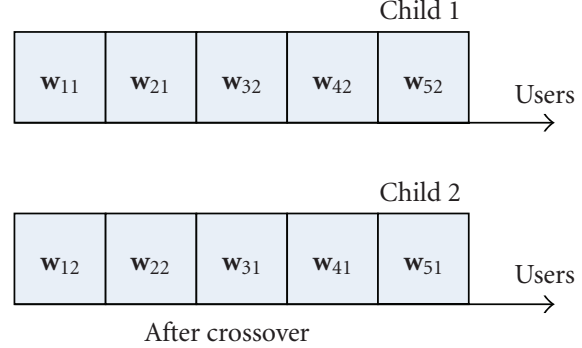
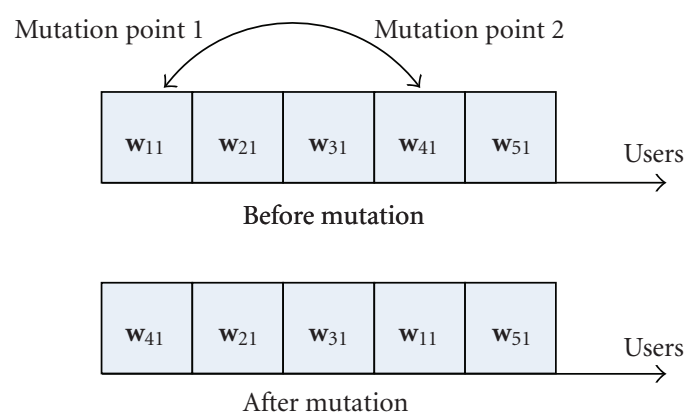

FIgURE 14: Crossover and mutation operations in genetic algorithm.

Maximising $Q_{d}$, as defined in (35), is equivalent to minimise $\varphi(\mathbf{w})$ as $Q(x)$ is a decreasing function of $x$, where $\varphi(\mathbf{w})$ is given by

$$
\begin{aligned}
\varphi(\mathbf{w})= & \frac{\sqrt{\mathbf{w}^{T}\left[2 N \mathbf{G}^{2} \boldsymbol{\Sigma}^{2}+\Delta\right] \mathbf{w}} Q^{-1}\left(Q_{f}\right)}{\sqrt{\mathbf{w}^{T}\left[2(N \mathbf{I}+2 \boldsymbol{\Gamma}) \mathbf{G}^{2} \Sigma^{2}+\Delta\right] \mathbf{w}}} \\
& +\frac{N \mathbf{g}^{T} \boldsymbol{\Sigma} \mathbf{w}-\mathbf{g}^{T}((N \mathbf{I}+\boldsymbol{\Gamma}) \odot \boldsymbol{\sigma}) \mathbf{w}}{\sqrt{\mathbf{w}^{T}\left[2(N \mathbf{I}+2 \boldsymbol{\Gamma}) \mathbf{G}^{2} \boldsymbol{\Sigma}^{2}+\Delta\right] \mathbf{w}}} \\
= & \frac{\sqrt{\mathbf{w}^{T}\left[2 N \mathbf{G}^{2} \boldsymbol{\Sigma}^{2}+\Delta\right] \mathbf{w} Q^{-1}}\left(Q_{f}\right)-\Gamma \mathbf{g}^{T} \mathbf{\Sigma} \mathbf{w}}{\sqrt{\mathbf{w}^{T}\left[2(N \mathbf{I}+2 \boldsymbol{\Gamma}) \mathbf{G}^{2} \boldsymbol{\Sigma}^{2}+\Delta\right] \mathbf{w}}} .
\end{aligned}
$$

Similarly for fading channels, the average probability of detection can be obtained by averaging $Q_{d}$ over fading statistics as described in Section 3.2. Now the optimisation problem can be formulated as

$$
\begin{aligned}
\operatorname{minimise} & \varphi(\mathbf{w}) \\
\text { s.t. } & \sum_{i=1}^{M} w_{i}=1, \quad w_{i} \geq 0, \forall i \in\{1,2,3, \ldots, M\} .
\end{aligned}
$$

6.1. GA-Based Weighted Collaborative Spectrum Sensing. This section describes the design of a GA-based weighted CSS framework for the case of SDC at the fusion centre. In this work, GA is used as a solution approach to minimise $\varphi(\mathbf{w})$ as defined in (36) for a given value of $Q_{f}$. The GA has been proposed as a computational analogy of adaptive systems by Holland [54]. They are modelled based on the principles of natural evolution and selection and is briefly described in this section. An initial population is first generated and then the fitness of each chromosome in the initial population is evaluated using a predefined fitness function. A loop is initiated to simulate the generations and in each generation, chromosomes are selected probabilistically according to their fitness. The genes of the selected individuals will mutate and crossover to produce offsprings to maintain the population size. The GA continues to iterate until the convergence is achieved or until it exceeds the maximum number of generations.

6.1.1. Seeding. The algorithm starts by randomly generating an initial population of possible solutions. Here, the initial population is the randomly generated values of weights satisfying the constraints as described in (37). Seeding is a process of setting the initial population to some initial configuration. If the initial population is seeded properly, the performance of GA can be greatly enhanced. Since GA works by probabilistically mutating and combining, the convergence of algorithm can be achieved quickly if the population is initially preset to a good solution.

6.1.2. Fitness Function. A fitness function plays a central role in GA. It evaluates fitness of each chromosome and forces the algorithm to search for optimal solutions and is the only link between actual problem and the GA. A fitness function ranks chromosomes in a given population; so individuals having better fitness values have higher chances of survival and reproduction in the next generation. In this paper, 


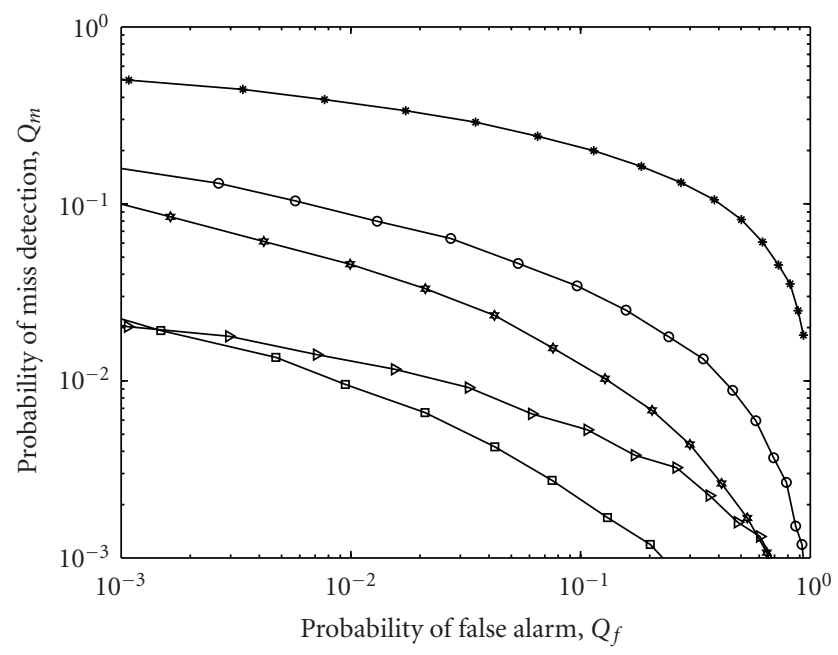

$\rightarrow M=1$, EGC (same SNR) $\rightarrow M=6$, EGC (different SNR)
$\rightarrow M=3$, EGC (same SNR) $\rightarrow M=6$, PC (different SNR)
$\rightarrow M=6$, EGC (same SNR)

FIGURE 15: Receiver operating characteristics for collaborative spectrum sensing with perfect reporting channel, $N=10$.

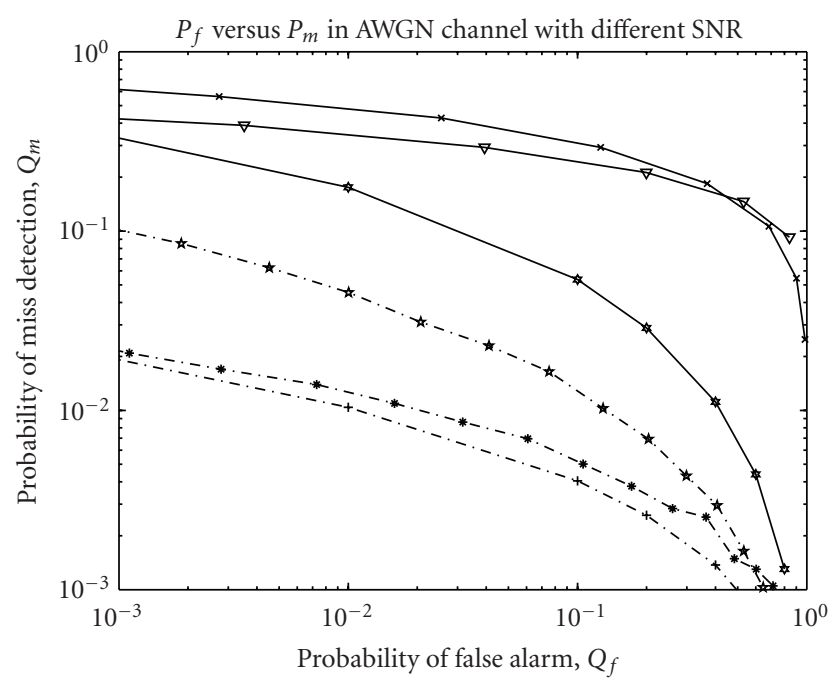

$$
\begin{aligned}
& \text { * - EGC, no channel gains } \\
& \rightarrow \text { - SC, no channel gains } \\
& \rightarrow \text { EGC, with channel gains }
\end{aligned}
$$

FIGURE 16: Receiver operating characteristics for collaborative spectrum sensing for 6 users in AWGN channel with im-perfect reporting channels, $N=10$.

$\varphi(\mathbf{w})$ is used as a fitness function to evaluate the fitness of individuals. After calculating the fitness of each individual, all fitness values are scaled in a range that is suitable for the selection algorithm. The selection algorithm uses these scaled fitness values to choose the parents of the next generation. The range of scaled values affects the performance of GA, and in this paper the scaling method described by Goldberg [55] is used.

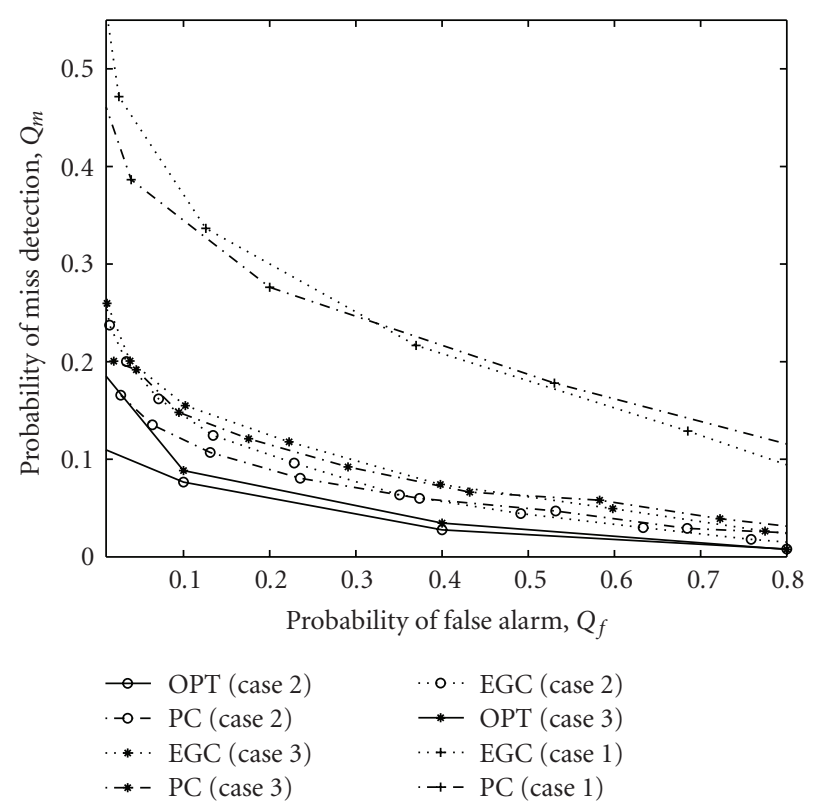

FIGURE 17: Receiver operating characteristics for collaborative spectrum sensing for 6 users in Rayleigh fading channel with imperfect reporting channels, $N=10$ (Case 1: All users have good reporting channel, Case 2: All users have bad reporting channel, and Case 3: Two of the users have good reporting channel).

6.1.3. Selection. Once the chromosomes in a given population have been evaluated according to their fitness values, the one with the better fitness will be selected, and the others will be eliminated. There are many different strategies available in the literature to implement selection algorithm [56]. The simplest and the most widely used selection scheme, the roulette wheel selection, is used in simulations [55].

6.1.4. Elitism. The number of chromosomes in a population with the best-scaled fitness value guaranteed to survive in the next generation and represented by Elitism is called Elite children. Proper value of Elite children is important in the fast convergence of GA.

6.1.5. Crossover and Mutation. Crossover process in GA combines two individuals (parents) and produces entirely new chromosomes (children). The main idea behind crossover operation is that the children may be better than both of the parents if they take the best attributes from each parent. Generally, crossover occurs during evolution according to a specified probability and is typically in the range of $80 \%$ to $90 \%$. Although a number of crossover techniques are available in the literature, the simplest crossover technique, called single-point crossover [56], is used in this study.

Mutation is another genetic operation which alters one or more genes in a chromosome from its original state. This introduces new genetic material in the population. With the new gene, GA may be able to arrive at a better solution than previously possible. Mutation also occurs during the evolution process by some prespecified probability and this value is normally small as compared to the crossover 
TABLE 1: GA parameter configuration.

\begin{tabular}{lc}
\hline Parameter & Value \\
\hline Population Size & 100 \\
Number of Generations & 40 \\
Elitism & $2 \%$ \\
Mutation Probability & $2 \%$ \\
Crossover Probability & $80 \%$ \\
Initialisation Method & Random \\
Crossover operation & Single point \\
Selection Method & Roulette wheel \\
\hline
\end{tabular}

probability. In the classical mutation process, one or more pairs of genes are selected randomly and swapped to produce new offsprings. Figure 14 illustrates the process of crossover and mutation for the case of 5 collaborating users.

6.1.6. Termination. Termination is the criteria by which GA decides whether to continue or stop searching for better solutions. There are many possibilities to terminate GA including generation number, evolution time, and population convergence, and so forth and in this work generation number criterion is used for terminating the GA. Based on a number of test experiments, the best suited GA parameter configuration was set up for the optimisation problem and parameters are listed in Table 1.

6.2. Numerical Result and Discussions. In this section, proposed GA-based weighted CSS scheme for SDC is simulated and compared with existing weighting schemes proposed in [42], that is, Equal Gain Combining (EGC) and Proportional Combining (PC). EGC is the weighting scheme in which all the collaborating SUs have equal weights and in PC the fusion centre assigns proportional weight to SUs according to their SNR values. Numerical results are obtained from simulations for use Case 2 over 1,000,000 noise realisations for the given set of noise variances. Noise variance of all collaborating users for the primary channel (i.e., channel between primary transmitter and secondary users) is assumed to be $\sigma^{2}=1$ and noise variance of the reporting channels is assumed to be $\delta^{2}=1 \mathrm{~dB}$. Value of $N$ is assumed to be 10 in all simulations.

Figure 15 shows the probability of miss detection $Q_{m}$ against probability of false alarm $Q_{f}$ with different number of collaborating users and their corresponding SNR values. A perfect reporting channel is assumed here and the channel between SUs and PU is considered to be AWGN channel. Figure 15 shows clearly that with an increase in the number of collaborating users sensing performance improves if all SUs have same SNR. However, when the cognitive users have different mean SNR values, then the sensing performance degrades with equal gain combining. Proportional weights assigned to different users according to their SNR values improve sensing performance as compared to equal gain combining approach. From the results it is concluded that users SNRs have a direct impact on the spectrum sensing performance.

Figure 16 plots the $Q_{m}$ versus $Q_{f}$ for the case when cognitive users have different SNRs and the reporting channel is not perfect; that is, practical AWGN channels exist between SUs and the fusion centre with different channel gains defined as $\mathbf{g}=[0.32,0.2,0.2,0.1,0.3,0.15]^{T}$. The value of channel gain is dependent on the location of the fusion centre and the SU and is varying over time. It can be seen from Figure 16 that reporting channel gains degrade the performance of spectrum sensing. Without channel gains, PC performs better than EGC, but, in the presence of reporting channel, PC does not perform much better than EGC. This is mainly because of the fact that in the presence of imperfect reporting channel, optimum weights of cognitive users are not only dependant on SNR values but also depend on reporting channel conditions. Under such conditions an analytical expression for the probability of detection is derived and optimum weights are calculated using GA. The result shows that the proposed GA-based optimal weights, denoted as "OPT," yield superior spectrum sensing performance in both cases, that is, with and without reporting channel gain.

In order to evaluate the performance of proposed optimised collaborative spectrum sensing framework, performance of GA-based optimisation algorithm is tested in fading channel. Three different cases were considered: Case 1 refers to the case in which all the SUs have good reporting channel, Case 2 is the case in which all the collaborating cognitive users have bad reporting channel, while in Case 3 two of the collaborating users have strong channel, while others have bad reporting channel. As seen from Figure 17 spectrum sensing performance is the worst for Case 1 and the best for Case 3; however, in all of the three cases, the performance of the proposed optimised thresholds outperforms the other solutions.

\section{Conclusions}

Spectrum is a scarce resource and it has been a major focus of research over the last several decades. Cognitive radio technology, which is a one of the promising approaches to utilise radio spectrum efficiently, has become an attractive option. Deployment of cognitive radio networks mainly depends on the ability of cognitive devices to detect licensed or primary users accurately and hence minimise interference to the licensed users. Spectrum sensing has been identified as a key functionality of a cognitive radio. However, as observations of a single cognitive radio are not always trustworthy, so collaboration of cognitive users is normally required to improve licensed users detection performance. In this paper, optimisation algorithms for both hard decision and soft decision combining are presented for collaborative spectrum sensing. It is well known that fusion strategy at the fusion centre has direct impact on the overall performance of collaborative spectrum sensing. We consider optimisation of both hard and soft decision fusion and develop algorithms to optimise spectrum sensing performance. It is concluded 
that in order to derive an optimum fusion rule, the fusion centre must know the collaborating users estimated SNR values, channel conditions, as well as their 1-bit decision for the case of hard decision fusion. We also proposed a genetic algorithm-based optimisation of weighted collaborative spectrum sensing in which weights are assigned to the information provided by the users to improve CSS in terms of ROC. The optimum weight vector is obtained by maximising the global probability of detection at the fusion centre. Simulation results show that the proposed strategies improve spectrum sensing performance in terms of global probability of miss detection. However, proposed schemes require knowledge about SNR of all users, channel conditions, reporting channel gains, and so forth which need larger bandwidths. Our future research will consider efficient protocols and techniques to optimise bandwidth utilisation for the cases presented in this paper.

\section{Appendices}

\section{A. Derivation of Probability of False Alarm for Energy Detector}

The probability distribution function of a chi-square random variable $X$ with $2 N$ degrees of freedom is given by

$$
f_{X}(x)=\frac{x^{N-1} e^{-x / 2}}{2^{N} \Gamma(N)}
$$

where $\Gamma(\cdot)$ is gamma function and is defined as

$$
\Gamma(u)=\int_{0}^{\infty} a^{u-1} e^{-t} d t
$$

Now for a given threshold $\lambda$ the probability of false alarm under hypothesis $\mathscr{H}_{0}$ (as defined in (1)) can be computed as

$$
\begin{aligned}
P_{f} & =\operatorname{Prob}\left\{X>\lambda \mid \mathscr{H}_{0}\right\} \\
& =\int_{\lambda}^{\infty} f_{X}(x) d x \\
& =\int_{\lambda}^{\infty} \frac{x^{N-1} e^{-x / 2}}{2^{N} \Gamma(N)} d x .
\end{aligned}
$$

Let $x=2 u$; so,

$$
\begin{aligned}
P_{f} & =\frac{1}{2^{N} \Gamma(N)} \int_{\lambda / 2}^{\infty} 2^{N-1} u^{N-1} e^{-u} 2 d u \\
& =\frac{1}{\Gamma(N)} \int_{\lambda / 2}^{\infty} u^{N-1} e^{-u} d u .
\end{aligned}
$$

From the definition of incomplete gamma function $\Gamma(s, x)=$ $\int_{x}^{\infty} t^{s-1} e^{-t} d t$

$$
P_{f}=\frac{\Gamma(N, \lambda / 2)}{\Gamma(N)} .
$$

\section{B. Derivation of Probability of Detection for Energy Detector}

Probability density function of noncentral chi-square random variable $x$ with $2 \mathrm{~N}$ degrees of freedom and noncentrality parameter of $2 \mathrm{~N} \gamma$ is given by

$$
f_{X}(x)=\frac{1}{2}\left(\frac{x}{2 N \gamma}\right)^{(N-1) / 2} \exp \left(-\frac{x+2 N \gamma}{2}\right) I_{N-1}(\sqrt{2 N \gamma x}) .
$$

So for the threshold $\lambda$, probability of detection, that is, probability that $X>\lambda$ under $\mathscr{H}_{1}$, is given as

$$
\begin{aligned}
P_{d} & =\operatorname{Prob}\left\{X>\lambda \mid \mathscr{H}_{1}\right\} \\
& =\int_{\lambda}^{\infty} f_{X}(x) d x \\
& =\int_{\lambda}^{\infty} \frac{1}{2}\left(\frac{x}{2 N \gamma}\right)^{(N-1) / 2} \exp \left(-\frac{x+2 N \gamma}{2}\right) I_{N-1}(\sqrt{2 N \gamma x}) d x .
\end{aligned}
$$

Assume $x=z^{2}$; then,

$$
\begin{aligned}
P_{d}= & \int_{\sqrt{\lambda}}^{\infty} \frac{1}{2}\left(\frac{z^{2}}{2 N \gamma}\right)^{(N-1) / 2} \\
& \times \exp \left[-\frac{z^{2}+2 N \gamma}{2}\right] I_{N-1}(z \sqrt{2 N \gamma}) 2 z d z \\
= & \int_{\sqrt{\lambda}}^{\infty} \frac{1}{(2 N \gamma)^{(N-1) / 2} z \cdot z^{N-1}} \\
& \times \exp \left[-\frac{z^{2}+(\sqrt{2 N \gamma})^{2}}{2}\right] I_{N-1}(z \sqrt{2 N \gamma}) d z .
\end{aligned}
$$

Using definition of generalised Marcum Q-function,

$$
Q_{m}(\alpha, \beta)=\frac{1}{\alpha^{m-1}} \int_{\beta}^{\alpha} x^{m} \exp \left[-\frac{x^{2}+\alpha^{2}}{2}\right] I_{m-1}(\sqrt{\alpha x}) d x .
$$

$P_{d}$ can be expressed in terms of generalised Marcum $Q$ function, with $m=N, x=z, \alpha=\sqrt{2 N \gamma}$, and $\beta=\sqrt{\lambda}$, as

$$
P_{d}=Q_{N}(\sqrt{2 N \gamma}, \sqrt{\lambda})
$$

\section{Acknowledgments}

This work was performed in the project E3 which has received research funding from the EU FP7 framework. This paper reflects only the authors views and the community is not liable for any use that may be made of the information contained therein. The contributions of colleagues from the E3 consortium are hereby acknowledged. 


\section{References}

[1] "International Telecommunication Union: Radio Regulations Revised," 1994.

[2] "Technology Research Programme: Research and Development at Ofcom 2004/05," October, p.37, 2005, http://www.ofcom.org.uk/research/technology/overview/techrandd200405/.

[3] "Federal Communications Commission: Spectrum policy task force report," November 2002, http://fjallfoss.fcc.gov/.

[4] A. Shukla, "Cognitive radio technology—a study for Ofcom," Tech. Rep. 830000143, QinetiQ Ltd, Hampshire, UK, 2006.

[5] M. McHenry, E. Livsics, T. Nguyen, and N. Majumdar, "XG dynamic spectrum sharing field test results," in Proceedings of the 2nd IEEE International Symposium on New Frontiers in Dynamic Spectrum Access Networks, pp. 676-684, May 2007.

[6] Spectrum Management Advisory Group (SMAG), "Position Paper on UMTS," March 2009, http://www.ofcom .org.uk/static/archive/ra/smag/papers/umts.htm.

[7] P. Leaves, Dynamic spectrum allocation between cellular and broadcast systems, Ph.D. dissertation, University of Surrey, 2004.

[8] “FCC Auctions March 2008," July 2009, http://wireless .fcc.gov/.

[9] A. Ghasemi and E. S. Sousa, "Opportunistic spectrum access in fading channels through collaborative sensing," IEEE Journal of Communications, vol. 2, no. 2, pp. 71-81, 2007.

[10] T. Yücek and H. Arslan, "A survey of spectrum sensing algorithms for cognitive radio applications," IEEE Communications Surveys and Tutorials, vol. 11, no. 1, pp. 116-130, 2009.

[11] H. Urkowitz, "Energy detection of unknown deterministic signals," IEEE Proceedings, vol. 55, no. 4, pp. 523-531, 1967.

[12] A. Sonnenschein and P. M. Fishman, "Radiometric detection of spread-spectrum signals in noise of uncertain power," IEEE Transactions on Aerospace and Electronic Systems, vol. 28, no. 3, pp. 654-660, 1992.

[13] Federal Communications Commission, "Notice of Proposed Rule Making, in the matter of unlicensed operaion in TV broadcast bands," May 2004, http://www.fcc.gov/.

[14] IEEE802.22, "Working Group on Wireless Regional Area Networks (WRAN)," Tech. Rep., IEEE, New York, NY, USA, 2009.

[15] Ofcom, "Digital Divident Review, A statement and our approach to awarding the digital divident," December 2007, http://www.ofcom.org.uk/consult/condocs/ddr/.

[16] DRIVE Project Web Site, March 2009, http://www.istdrive.org/index $2 . h$ tml.

[17] Over-DRiVE Project Web Site, March 2009, http://www.istoverdrive.org/.

[18] WINNER Project Web Site, March 2009, https://www.istwinner.org.

[19] E2R II Project Web Site, March 2009, E2R II Project.

[20] ORACLE Project Web Site, March 2009, http://www.istoracle.org/.

[21] E3 Project Web Site, March 2009, https://ict-e3.eu/.

[22] Radio Access \& Spectrum Cluster Web Site, March 2009, http://www.newcom-project.eu:8080/Plone/ras.

[23] DARPA neXt Generation (XG) Communications Working Group, March 2009, http://www.ir.bbn.com/projects/xmac/ working-group/index.html.

[24] National Science Foundation, "Networking Technology and Systems (NeTS) Program Solicitation," March 2009, http://www.nsf.gov/pubs/2005/nsf05505/nsf05505.htm.

[25] P. Demestichas, A. Katidiotis, K. A. Tsagkaris, E. F. Adamopoulou, and K. P. Demestichas, "Enhancing channel estimation in cognitive radio systems by means of Bayesian networks," Wireless Personal Communications, vol. 49, no. 1, pp. 87-105, 2008.

[26] P. Demestichas, "Enhanced network selections in a cognitive wireless B3G world," Annals of Telecommunications, vol. 64, no. 7-8, pp. 483-501, 2009.

[27] Z. Xueqiang, C. U. I. Li, C. Juan, W. U. Qihui, and W. Jinlong, "Cooperative spectrum sensing in cognitive radio systems," in Proceedings of the 1st International Congress on Image and Signal Processing (CISP '08), pp. 262-266, June 2008.

[28] W. Wang, W. Zou, Z. Zhou, H. Zhang, and Y. Ye, "Decision fusion of cooperative spectrum sensing for cognitive radio under bandwidth constraints," in Proceedings of the 3rd International Conference on Convergence and Hybrid Information Technology (ICCIT '08), pp. 733-736, December 2008.

[29] W. Zhang, R. K. Mallik, and K. Ben Letaief, "Cooperative spectrum sensing optimization in cognitive radio networks," in Proceedings of IEEE International Conference on Communications (ICC '08), pp. 3411-3415, cn, June 2008.

[30] X. Chen, Z. s. Bie, and W. 1. Wu, "Detection efficiency of cooperative spectrum sensing in cognitive radio network," Journal of China Universities of Posts and Telecommunications, vol. 15, no. 3, pp. 1-7, 2008.

[31] T. Aysal, S. Kandeepan, and R. Piesiewicz, "Cooperative spectrum sensing over imperfect channels," in Proceedings of IEEE Globecom Workshops (GLOBECOM '08), pp. 1-5, December 2008.

[32] K. Arshad and K. Moessner, "Collaborative spectrum sensing for cognitive radio," in Proceedings of IEEE International Conference on Communications Workshops (ICC '09), pp. 1-5, June 2009.

[33] E. Peh, Y. C. Liang, E. Peh, and Y. C. Liang, "Optimization for cooperative sensing in cognitive radio networks," in Proceedings of IEEE Wireless Communications and Networking Conference (WCNC '07), pp. 27-32, April 2007.

[34] D. J. Kadhim, S. Gong, W. Liu, and W. Cheng, "Optimization of Cooperation sensing spectrum performance," in Proceedings of WRI International Conference on Communications and Mobile Computing (CMC '09), pp. 78-82, cn, February 2009.

[35] E. Peh, Y.-C. Liang, and Y. L. Guan, "Optimization of cooperative sensing in cognitive radio networks: a sensingthroughput tradeoff view," in Proceedings of IEEE International Conference on Communications, June 2009.

[36] C. Sun, W. Zhang, and K. Letaief, "Cluster-based cooperative spectrum sensing in cognitive radio systems," in Proceedings of IEEE International Conference on Communications (ICC '07), pp. 2511-2515, June 2007.

[37] Z. Chair and P. K. Varshney, "Optimal data fusion in multiple sensor detection systems," IEEE Transactions on Aerospace and Electronic Systems, vol. 22, no. 1, pp. 98-101, 1986.

[38] F. F. Digham, M. S. Alouini, and M. K. Simon, "On the energy detection of unknown signals over fading channels," IEEE Transactions on Communications, vol. 55, no. 1, pp. 21-24, 2007.

[39] W. Wang, L. Zhang, W. Zou, and Z. Zhou, "On the distributed cooperative spectrum sensing for cognitive radio," in Proceedings of International Symposium on Communications and Information Technologies (ISCIT '07), pp. 1496-1501, November 2007.

[40] E. Visotsky, S. Kuffher, and R. Peterson, "On collaborative detection of TV transmissions in support of dynamic spectrum sharing," in Proceedings of the 1st IEEE International Symposium on New Frontiers in Dynamic Spectrum Access Networks (DySPAN '05), pp. 338-345, December 2005. 
[41] S. M. Mishra, A. Sahai, and R. W. Brodersen, "Cooperative sensing among cognitive radios," in Proceedings of IEEE International Conference on Communications (ICC '06), pp. 1658-1663, August 2006.

[42] F. E. Visser, G. J. M. Janssen, and P. Pawełczak, "Multinode spectrum sensing based on energy detection for dynamic spectrum access," in Proceedings of the 67th IEEE Vehicular Technology Conference (VTC '08), pp. 1394-1398, June 2008.

[43] Z. Quan, S. Cui, and A. Sayed, "Optimal linear cooperation for spectrum sensing in cognitive radio networks," IEEE Journal on Selected Topics in Signal Processing, vol. 2, no. 1, pp. 28-40, 2008.

[44] S. Haykin, "Cognitive radio: brain-empowered wireless communications," IEEE Journal on Selected Areas in Communications, vol. 23, no. 2, pp. 201-220, 2005.

[45] A. Goldsmith, Wireless Communications, Cambridge University Press, New York, NY, USA, 2005.

[46] P. K. Varshney, Distributed Detection and Data Fusion, Springer, Secaucus, NJ, USA, 1996.

[47] R. F. Mills and G. E. Prescott, "A comparison of various radiometer detection models," IEEE Transactions on Aerospace and Electronic Systems, vol. 32, no. 1, pp. 467-473, 1996.

[48] I. Akyildiz, W.-Y. Lee, M. C. Vuran, and S. Mohanty, "A survey on spectrum management in cognitive radio networks," IEEE Communications Magazine, vol. 46, no. 4, pp. 40-48, 2008.

[49] T. Weiss, J. Hillenbrand, and F. Jondral, "A diversity approach for the detection of idle resources in spectrum pooling systems," in Proceedings of the 48th International Scientific Colloquium, pp. 37-38, September 2003.

[50] C. Da Silva, B. Choi, and K. Kim, "Distributed spectrum sensing for cognitive radio systems," in Proceedings of the Information Theory and Applications Workshop (ITA '07), pp. 120-123, March 2007.

[51] A. K. Kattepur, A. T. Hoang, Y.-C. Liang, and M. J. Er, "Data and decision fusion for distributed spectrum sensing in cognitive radio networks," in Proceedings of the 6th International Conference on Information, Communications and Signal Processing (ICICS '07), pp. 1-5, December 2007.

[52] M. Gudmundson, "Correlation model for shadow fading in mobile radio systems," Electronics Letters, vol. 27, no. 23, pp. 2145-2146, 1991.

[53] R. A. Horn and C. R. Johnson, Matrix Analysis, Cambridge University Press, Cambridge, UK, 1985.

[54] J. H. Holland, Adaptation in Natural and Artificial Systems: An Introductory Analysis with Applications to Biology, Control, and Artificial Intelligence, MIT Press, Cambridge, Mass, USA, 1992.

[55] D. E. Goldberg, Genetic Algorithms in Search, Optimization and Machine Learning, Addison-Wesley Longman, Boston, Mass, USA, 1989.

[56] M. Gen and R. Cheng, Genetic Algorithms and Engineering Design, Wiley, New York, NY, USA, 1997. 

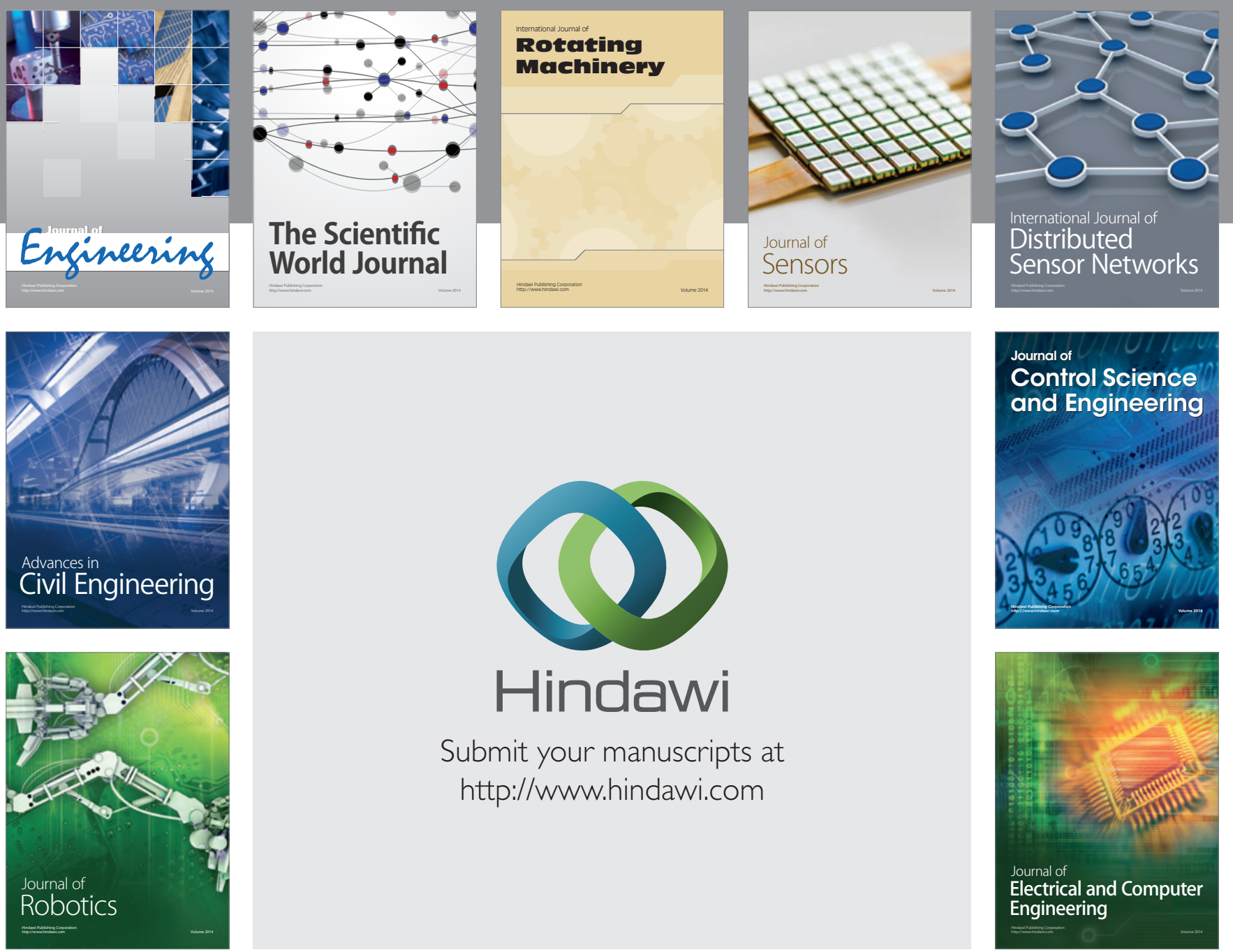

Submit your manuscripts at

http://www.hindawi.com
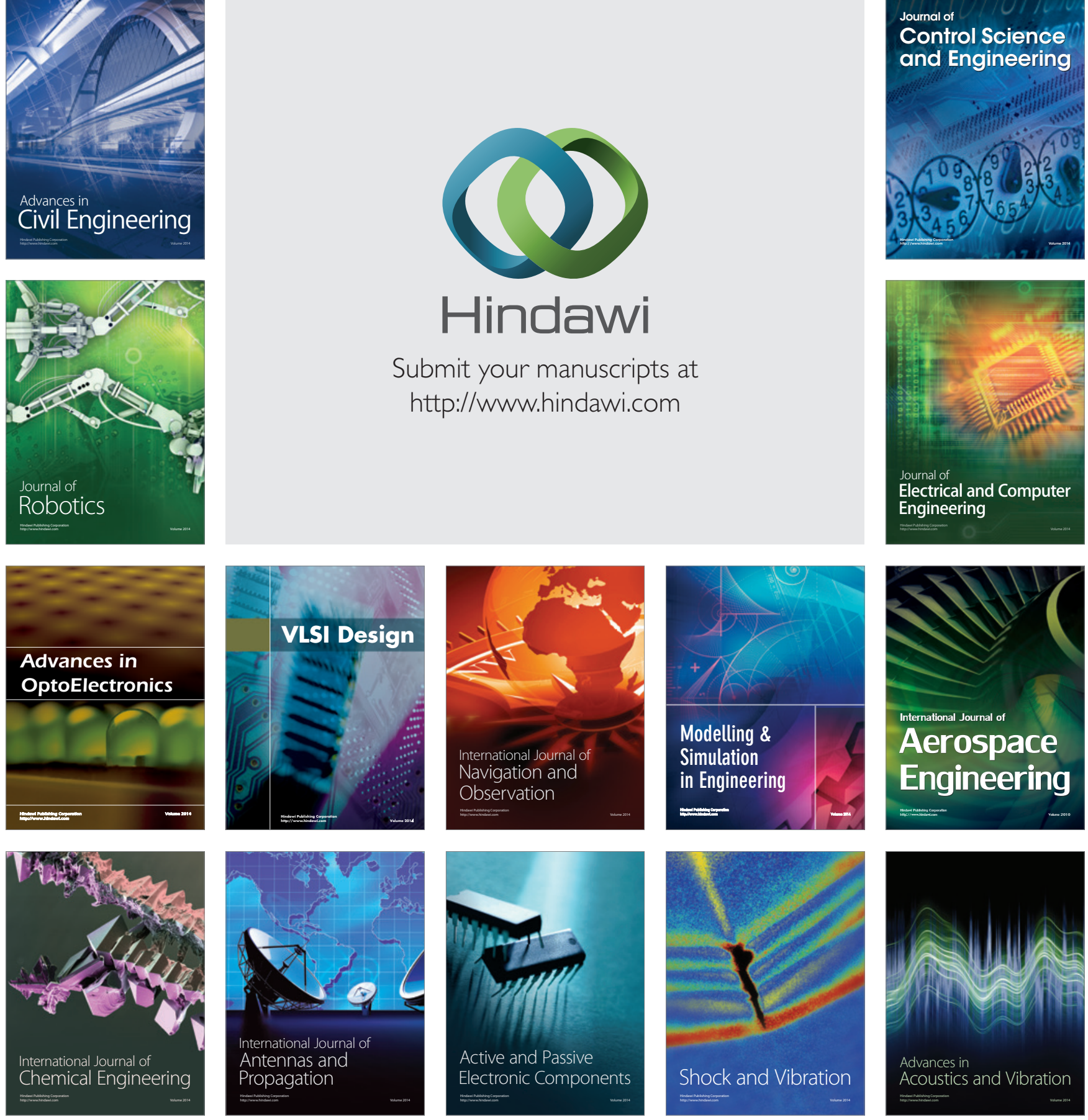